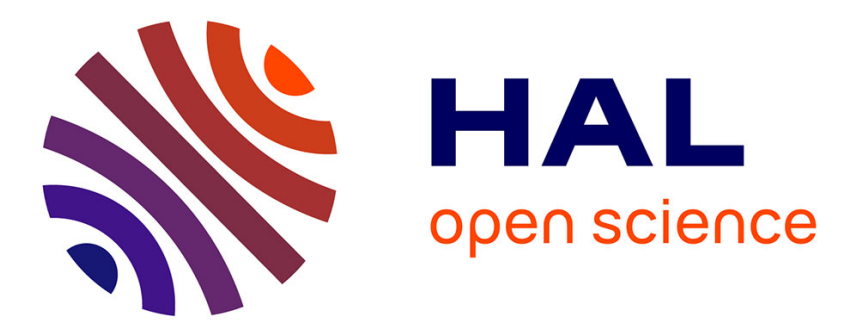

\title{
Les effets des allégements de cotisations sociales sur l'emploi et les salaires: Une évaluation de la réforme de 2003
}

\author{
Mathieu Bunel, Fabrice Gilles, Yannick L'Horty
}

\section{- To cite this version: \\ Mathieu Bunel, Fabrice Gilles, Yannick L'Horty. Les effets des allégements de cotisations sociales sur l'emploi et les salaires: Une évaluation de la réforme de 2003. Economie et Statistique / Economics and Statistics, 2009, 429-430, pp.77-105. halshs-00658475}

\section{HAL Id: halshs-00658475 \\ https://shs.hal.science/halshs-00658475}

Submitted on 10 Jan 2012

HAL is a multi-disciplinary open access archive for the deposit and dissemination of scientific research documents, whether they are published or not. The documents may come from teaching and research institutions in France or abroad, or from public or private research centers.
L'archive ouverte pluridisciplinaire HAL, est destinée au dépôt et à la diffusion de documents scientifiques de niveau recherche, publiés ou non, émanant des établissements d'enseignement et de recherche français ou étrangers, des laboratoires publics ou privés. 


\title{
Les effets des allégements de cotisations sociales sur l'emploi et les salaires : Une évaluation de la réforme de 2003
}

\author{
Matthieu Bunel*, Fabrice Gilles**, Yannick L'Horty***
}

\begin{abstract}
La réforme du 17 janvier 2003 a unifié les dispositifs d'exonération de cotisations sociales entre les entreprises à 35 heures et celles à 39 heures tout en réalisant sur une période de trois ans, de 2003 à 2005, la convergence entre le Smic horaire et les différentes garanties mensuelles de rémunération qui coexistaient jusque-là. Nous évaluons les effets de cette réforme sur l'emploi et les salaires au moyen d'un appariement de données issues des fichiers de l'Urssaf et de l'Insee. Ces données permettent, pour la première fois, d'appréhender les baisses de cotisations sociales effectivement perçues par les entreprises.
\end{abstract}

L'évaluation tient compte à la fois de l'endogénéité de la baisse du coût du travail induite par la réforme (le coût du travail diminue davantage dans les entreprises qui ont fait une plus large place aux emplois à bas salaires à la suite de la réforme) et des différences entre les entreprises initialement à 35 heures ou à 39 heures. La variable clé est la variation du coût du travail directement liée aux changements de barèmes et à la hausse des minima salariaux (indépendamment des ajustements opérés par l'entreprise après 2002). Elle est calculée pour chaque entreprise sur la base des rémunérations versées en 2002 et des hausses du salaire minimum. Les effets sur l'emploi et les salaires de cette variation sont ensuite estimés à l'aide de méthodes paramétriques et semi-paramétriques. Selon ces estimations, la réforme de 2003 a permis d'accroître légèrement l'emploi dans les entreprises restées à 39 heures mais elle a conduit à l'effet inverse pour les entreprises à 35 heures. Au total, son effet sur l'emploi total s'avère ambigu, qu'il soit mesuré en effectif ou en équivalent temps plein. Elle a cependant contribué à la hausse des rémunérations dans les deux catégories d'entreprises.

\footnotetext{
* Université de technologie de Belfort Montbéliard (Utbm), CEE et TEPP (FR CNRS n³126)

** Université de Lille 1, EQUIPPE et CEE

${ }^{* * *}$ ERUDITE, Université de Paris-Est, CEE et TEPP (FR CNRS $\left.n^{\circ} 3126\right)$

Ce travail a bénéficié du soutien de la Dares et s'inscrit dans un projet plus vaste réalisé au sein du centre d'études de l'emploi (CEE) auquel Richard Duhautois, Patrick Kwok, Marianne Pauchet et Corinne Perraudin ont également participé. Nous remercions l'Insee et l'Acoss pour la mise à disposition des données. Une première version de cet article a bénéficié des remarques et des suggestions de Dominique Goux, Alain Gubian, Nadia Halibay, Cyrille Hagneré, Stéphanie Jamet, Etienne Lehmann, Benoît Ourliac, Cyril Nouveau, Alain Trannoy, Elena Scancanelli, Henri Sterdyniak, ainsi que celles de deux rapporteurs anonymes et des participants aux journées LouisAndré Gérard Varet, JMA, AFSE, EALE, T2M et des séminaires de l'EPEE-Université d'Evry, du CLERSE-université de Lille 1, de l'Acoss, du CEE et de la Dares. Les erreurs et les omissions restent de la responsabilité des auteurs. Cet article n'exprime pas le point de vue des institutions citées.
} 
$\mathbf{L}$ a Loi $\mathrm{n}^{\circ} 2003-47 \mathrm{du} 17$ janvier 2003 relative « aux salaires, au temps de travail et au développement de l'emploi » a unifié les dispositifs d'exonération de cotisations sociales employeurs entre les entreprises à 35 heures et celles à 39 heures. Les exonérations atteignent alors un maximum, pour un montant de plus de 20 milliards d'euros à partir de 2004. Avec l'unification du Smic horaire et des Garanties mensuelles de rémunération (GMR) qu'elle organise également, cette réforme a fortement affecté l'évolution du coût du travail entre janvier 2003 et juillet 2005. Ces changements ont eu deux conséquences : d'une part une redistribution des exonérations de prélèvements sociaux entre les entreprises, avec moins d'exonérations données aux entreprises à 35 heures et davantage d'exonérations données aux entreprises aux 39 heures, d'autre part, au sein des entreprises, une redistribution de ces exonérations entre les salariés selon leur niveau de rémunération.

L'objectif de cet article est d'évaluer les effets de cette réforme sur l'emploi et les salaires. Pour ce faire, on assimile l'impact du nouveau dispositif aux effets d'un traitement dont on mesure les effets en cherchant à comparer les entreprises qui en ont bénéficié à celles qui n'en ont pas bénéficié. La difficulté de l'évaluation provient du fait que le traitement étudié affecte toutes les entreprises, de manière différenciée et endogène. Les entreprises qui ont le plus augmenté leur part de salariés dont la rémunération est comprise entre 1,2 Smic et 1,4 Smic ont bénéficié d'une baisse plus forte du coût du travail et donc d'un traitement d'une intensité plus forte a posteriori. Pour tenir compte de ces différences, on est amené à distinguer deux groupes d'entreprises. Le premier est constitué des entreprises, dites « Fillon-Aubry2 », qui bénéficiaient avant 2003 des allègements de cotisations sociales prévus dans le cadre des lois de juin 1998 et janvier 2000 associés au passage aux 35 heures, et qui ont obtenu ensuite les aides prévues par le dispositif Fillon. Le second groupe est constitué des entreprises, dites « Fillon-autres employeurs », restées aux 39 heures avant 2003, qui bénéficiaient pour leur très large majorité de la ristourne sur les bas salaires et qui ont ensuite obtenu les aides Fillon.

Pour ces deux groupes d'entreprises, on calcule l'ampleur de la variation du coût du travail liée à la hausse des salaires minimum et au changement des barèmes d'allègement en supposant stable la structure de leur main-d'œuvre avant et après la réforme de 2003. Cette stratégie s'inspire de celle retenue par Crépon et Desplatz (2001) pour analyser les effets de la politique d'allègements sur les bas salaires de
1995. Toutefois, notre méthodologie se différencie sur deux points.

D'une part, elle intègre les diverses modifications des barèmes d'allègement et la hausse différenciée des salaires minimum : le Smic horaire pour les entreprises restées aux 39 heures et les GMR pour les entreprises ayant mis en œuvre la réduction du temps de travail. D'autre part, elle se base sur les exonérations effectivement perçues par les entreprises et non sur les exonérations théoriques calculées comme le font la plupart des études existantes. L'écart entre ces deux grandeurs est susceptible d'introduire un biais pour erreur de mesure de grande ampleur (Stewart, 1983).

Un échantillon cylindré de près de 90000 entreprises issu de l'appariement les fichiers administratifs Arome, Orme, Sequoia, Suse et DADS gérés par l'Acoss et l'Insee est utilisé. On se focalise sur les entreprises de plus de cinq salariés issues du secteur privé non agricole, présentes dans l'échantillon sur la période 2002 à 2005 et bénéficiant des allègements « Fillon-Aubry 2 » et « Fillon-autres employeurs » de manière continue sur la période 2003 à 2004.

Plusieurs techniques économétriques paramétriques puis non paramétriques sont successivement utilisées afin de dégager des effets robustes. L'estimation de base s'appuie sur la méthode des moindres carrés ordinaires (MCO). Cette méthode vise à estimer l'effet moyen du traitement en supposant celui-ci exogène. On a également recours à des régressions quantiles pour tester l'impact du traitement sur l'ensemble de la distribution des variables d'intérêt. Finalement, pour lever l'hypothèse de relation linéaire entre les variables expliquées et les variables explicatives, ainsi que l'hypothèse de normalité des résidus, des estimations sont réalisées en utilisant les méthodes d'appariement sélectif sur le score de propension.

Après un rappel des résultats obtenus par des études empiriques antérieures, on présente l'évolution du coût du travail liée à la réforme Fillon. Mesurer l'évolution du taux d'exonération engendrée par la réforme de janvier 2003 et la variation du coût du travail lié exclusivement à la réforme Fillon sont deux étapes préliminaires et indispensables aux estimations proprement dites.

\section{Les études empiriques antérieures conduisent à des résultats nuancés}

Les effets attendus d'une reforme des barèmes de cotisations sociales employeurs dépendent 
de trois facteurs : la structure des allègements, la valeur prise par les élasticités de la demande et de l'offre de travail au coût du travail et l'impact des allègements sur les salaires. Ainsi, le degré de ciblage, la générosité et la pérennité des allègements de cotisations sociales employeurs influencent fortement l'efficacité des allègements (L'Horty, 2000 ; OCDE, 2003 ; Marx, 2005).

Plusieurs études théoriques ont mis en évidence les effets d'une politique de baisse de cotisations sociales sur les effectifs à bas salaires, sur le taux de chômage et les rémunérations en retenant différents cadres d'analyse : les modèles d'équilibre général, les modèles de concurrence monopolistique ou encore les modèles dynamiques (CSERC, 1996 ; Fitoussi, 2000). Les effets d'une politique de baisse des cotisations ont été simulés en calibrant ces modèles à l'aide de valeurs estimées de l'élasticité de la demande de travail à son coût (pour la France voir notamment l'article de Chéron, Hairault et Langot, 2008). La principale limite de ces évaluations a priori provient de la forte sensibilité des résultats aux valeurs utilisées pour calibrer le modèle. Toutefois, ces modèles permettent de bien spécifier les mécanismes économiques en présence (par exemple l'impact de ces réformes sur les salaires, la productivité et le volume de production).

Afin de compléter ces travaux, de nombreuses études appliquées ont analysé a posteriori les effets d'un changement opéré par un pays concernant les barèmes de cotisations sociales. La première génération d'études utilise des séries chronologiques (Brittain, 1971 ; Vroman, 1974a ; Beach et Balfour, 1983 ; Kugler et Kugler, 2008) ou se base sur des comparaisons internationales (Vroman, 1974b ; Bell et Nickell, 1997). Ces premiers travaux n'obtiennent pas de résultats concluants quant aux effets d'une modification des cotisations sociales sur l'emploi. Ces résultats ont largement été remis en cause ces dernières années en raison de leur forte dépendance aux variables omises. En effet, au niveau macro-économique des variables inobservées peuvent affecter simultanément les taux de cotisations observés d'une part, et les salaires et l'emploi d'autre part, biaisant ainsi les coefficients estimés.

Afin d'éviter ce problème, une seconde génération de travaux a eu recours à des données microéconomiques. Au moyen de données de panel américaines sur la période 1968 à 1974, Hamermesh (1979) montre qu'une hausse des cotisations sociales influence simultanément les salaires et l'emploi. Une augmentation de $1 \%$ des cotisations sociales s'accompagne en moyenne d'une baisse de $0,3 \%$ des salaires, le restant étant absorbé par une diminution de l'emploi.

En adoptant une méthodologie similaire, Gruber (1997) étudie l'influence globale du processus de privatisation du système de sécurité sociale chilien, mis en place en 1981, et qui s'est traduit par une forte baisse des cotisations sociales. À partir de données d'entreprises industrielles relatives à la période 1979 à 1986, il obtient que seuls les salaires sont affectés positivement par ce choc. L'effet sur l'emploi demeure non significatif.

Sur données françaises, les travaux de Kramarz et Philippon (2001) et de Crépon et Desplatz (2001) ont analysé l'impact de la baisse du coût du travail sur les bas salaires au cours des années 1990 liée à la réforme Juppé de 1995. $\mathrm{Au}$ moyen des données de l'enquête Emploi portant sur la période (1990-1998), Kramarz et Philippon (2001) analysent la probabilité de perdre son emploi pour deux groupes de salariés : ceux directement touchés par le changement de coût du travail et ceux qui ne le sont pas, mais qui se trouvent être les plus proches de la première catégorie dans la distribution des salaires. À l'aide des estimateurs en double différence, ils obtiennent qu'une hausse de $1 \%$ du coût du travail conduit à une augmentation de $1,5 \%$ de la probabilité de perdre son emploi. Avec les exonérations de cotisations sociales, la baisse du coût du travail au voisinage du salaire minimum exerce un effet positif sur l'emploi. Crépon et Desplatz (2001) utilisent quant à eux des données appariées salariés-employeurs sur la période 1994 à 1997 issues des Bénéfices réels normaux (BRN) et des Déclarations annuelles de données sociales (DADS) pour analyser les effets des réformes de 1995 et 1996 . Au terme d'estimations paramétriques et non paramétriques, ils concluent à une forte augmentation de l'emploi à la suite de la réforme (autour de 460000 emplois), sans tenir compte d'effets de bouclage macro-économique.

Afin de pallier à cette lacune, la dernière génération de travaux a recours à des données chronologiques au niveau sectoriel. Le principal avantage de cette démarche est de tenir compte des effets volume et de substitution intersectoriels. Lorsque l'on tient compte de ces effets (Jamet, 2005 ; Gafsi et al. 2005), l'impact sur l'emploi est sensiblement plus faible que celui identifié par Crépon et Desplatz (2001), en particulier pour les salariés à bas salaires. 


\section{L'évolution du coût du travail sur la période 2001 à 2005}

Sur la période 2001-2005, le coût du travail a fortement augmenté (Montaut, 2008). Cette évolution est la résultante de trois facteurs principaux :

- l'augmentation du Smic et de la GMR instaurée lors des lois sur la réduction du temps de travail ;

- la diffusion de la hausse de ces minima sur une partie des salaires, notamment ceux les plus proches du Smic ;

- la réforme des allègements de cotisations sociales patronales.

\section{GMR et Smic horaire}

Le salaire minimum de croissance instauré en 1970 est un seuil de salaire horaire. Il inclut le salaire de base, les avantages en nature et les majorations diverses ayant de fait le caractère d'un complément de salaire. Le montant du Smic était réévalué chaque $1^{\text {er }}$ juillet en fonction de la hausse des prix, de la moitié de la hausse réelle du salaire horaire ouvrier et d'éventuels « coups de pouce ».

Lors du changement de durée légale du travail avec le passage aux 35 heures en janvier 2000, le législateur a posé le principe d'une garantie d'évolution du pouvoir d'achat des salariés au Smic bénéficiant d'une réduction du temps de travail (RTT). La rémunération de ces salariés a été fixée en fonction de leur salaire mensuel avant la RTT. Ainsi la garantie mensuelle de rémunération (GMR) correspond au Smic horaire au moment du passage aux 35 heures multipliée par 169 heures (1). Les salariés à 35 heures bénéficiaient donc mécaniquement d'un salaire horaire plus élevé que le Smic horaire (2). Les cinq " générations » de GMR s'appliquent aux salariés passés aux 35 heures avant les $1^{\text {ers }}$ juillet 1999, 2000, 2001, 2002 et 2003.

Sur la période 1999-2002, ces GMR ont été revalorisées en fonction de la hausse des prix et de la moitié de la hausse du salaire mensuel ouvrier. Ainsi, avant la réforme de 2003, la progression des GMR était moindre que celle du Smic horaire afin de les faire converger à terme. L'objectif de la réforme de 2003 était, entre autres, d'accélérer ce processus pour faire converger par le haut, sur une période de trois ans, le Smic horaire et les différentes GMR.
Les résultats de ce processus sont présentés en annexe 1. Sur la période 2001-2005, le Smic horaire a augmenté de $20,4 \%$ en euros courants alors que les différentes GMR ont augmenté entre $8,0 \%$ et $12,6 \%$. La hausse du coût du travail générée par la hausse du Smic horaire et des GMR a donc été plus forte pour les entreprises restées aux 39 heures que pour les autres.

\section{La diffusion du Smic et des GMR}

Les salariés dont la rémunération est rattrapée par la nouvelle valeur de la GMR ou du Smic bénéficient mécaniquement d'une hausse de leur salaire. Entre $13 \%$ et $15 \%$ des salariés sont concernés par ce phénomène (Seguin, 2006). Par ailleurs, il existe également une diffusion de ces minima dans la grille des salaires (Koubi et LHommeau, 2007). Ainsi, les salariés dont la rémunération est située au voisinage des minima bénéficient le plus de leur revalorisation. En revanche, au-delà de 1,4 Smic, cet effet s'atténue fortement (augmentation inférieure à $5 \%$ de l'augmentation du Smic). La diffusion de la hausse des minima ne semble donc pas linéaire. Enfin, même si cette question n'a encore été explorée que par peu d'études, il est probable que la diffusion de la hausse du Smic horaire est plus importante que celle de la GMR, puisqu'elle est de plus grande ampleur et que les hausses de salaires étaient souvent encadrées par des accords collectifs dans les entreprises ayant adopté les 35 heures. D'après les études réalisées par la Dares, les trois quarts des salariés passés aux 35 heures avant 2000 et la moitié pour ceux passés en 2000 sont concernés par un gel ou une modération salariale d'une durée moyenne de deux ans (Gubian et alii, 2004).

\section{Réforme des allègements de cotisations sociales prévue par la loi de 2003}

$\mathrm{Au}$ terme de la réforme de 2003, un système unique d'allègement s'applique à toutes les entreprises. Ce système porte à $26 \%$ du salaire brut le montant de l'allègement pour un salarié rémunéré au Smic. Au-delà, ce montant est linéairement dégressif jusqu'à 1,6 Smic (cf. graphique I). Avant juillet 2003, deux systèmes d'allègements de cotisations patronales coexis-

\footnotetext{
1. La durée de 169 heures correspond à l'horaire mensuel d'un emploi à 39 heures hebdomadaires.

2. Pendant la période de convergence du Smic et des GMR, les entreprises passées aux 35 heures avaient la possibilité d'embaucher des salariés au Smic horaire 39 heures sous des conditions assez restrictives. Les données ne permettant pas d'identifier cette situation, nous avons supposé que les entreprises n'avaient pas recouru à cette possibilité.
} 
taient. Le premier dont bénéficiaient les entreprises passées aux 35 heures se caractérisait par un montant maximal d'allègement égal à $26 \%$ du Smic. Un barème dégressif jusqu'à $1,7 \mathrm{Smic}$ puis stable ensuite pour les autres salaires s'appliquait (cf. annexe 1, tableau B). Le second concernait les entreprises n'ayant pas adopté ce changement d'horaire et pouvant bénéficier de la « ristourne sur les bas salaires ", maximum avec un taux d'allègement de $18,6 \%$ du salaire pour une rémunération du niveau du Smic, et dégressive ensuite jusqu'à 1,3 Smic (cf. graphique I). La fusion de ces deux barèmes en un seul, opérée par la réforme Fillon, a eu lieu de façon progressive, en quatre étapes : en juillet 2003 et 2004, en janvier et juillet 2005.

Globalement, le supplément d'allègements de cotisations sociales employeurs engendré par la réforme de 2003 a été plus élevé pour les entreprises restées aux 39 heures que pour les autres. En outre, cette harmonisation par le haut des barèmes n'est valable que pour les salariés dont la rémunération est inférieure à $1,4 \mathrm{Smic}$. En effet, pour les entreprises passées aux 35 heures, la réforme a conduit à une diminution, voire à une suppression des allègements octroyés jusque-là. Cet avantage relatif donné aux entreprises aux 39 heures visait à contrebalancer la hausse du
Smic horaire brut. Toutefois, même en tenant compte de cette hausse du Smic, la réforme n'a pas été neutre pour le coût du travail.

\section{Deux entreprises bénéficiaires sur trois encore aux 39 heures en 2003}

L'échantillon utilisé dans cette étude provient de l'appariement des fichiers de l'Acoss (Agence centrale des organismes de sécurité sociale) et de l'Insee. Les caractéristiques de ces données sont détaillées dans l'encadré 1 .

Cette base de données permet de décrire la population des entreprises étudiées (cf. annexe 2). $65 \%$ étaient aux 39 heures avant 2003 et ont bénéficié des exonérations "Fillon autres employeurs " et $35 \%$ étaient passées aux 35 heures et bénéficiaient des exonérations « Fillon-Aubry 2 ». Les entreprises à 35 heures disposent plus souvent d'un effectif inférieur à 20 salariés ( $73 \%$ contre $56 \%$ ), appartiennent moins souvent à un groupe et au secteur industriel (écarts respectifs de 10 et 5 points) et leur main-d'œuvre est moins féminisée ( $28 \%$ contre $38 \%$ ). Globalement, les entreprises de l'échantillon comportent 145000 établissements et emploient près de 3,4 millions de salariés. $83 \%$

\section{Graphique I}

\section{La fusion des dispositifs d'allègements de cotisations sociales}



Lecture : pour un niveau de salaire égal à 1,15 Smic, les cotisations patronales sont exonérées de 10 points de pourcentage dans le cadre du dispositif RBS (courbe en pointillés), de 18 points dans le cadre des allégements Aubry 2 (entreprises passées aux 35 heures avant 2003) et de 20 points dans le cadre du dernier dispositif Fillon au $1^{\text {er }}$ Juillet 2005.

Source : Légifrance. 
n'ont qu'un établissement. En ce qui concerne les autres (entreprises multi-établissements), très peu (moins de $1 \%$ ) se composent à la fois d'établissements ayant bénéficié des aides «Fillonautres employeurs » et d'établissements ayant obtenu des aides « Fillon-Aubry $2 »(3)$.

\section{Entre 2002 et 2005, les exonérations augmentent dans les entreprises à 39 heures...}

Le montant d'exonération par salarié au Smic était de $300 €$ par mois environ au premier semestre 2005 pour les deux catégories d'entreprises étudiées. De même, en 2005, le montant d'exonération moyen par salarié bénéficiant d'exonérations, c'est-à-dire rémunéré entre 1 et 1,7 Smic, était sensiblement le même pour les deux catégories d'entreprises (autour de $150 €$ par mois) (4).

En revanche, pour les salariés au Smic, les entreprises à 39 heures bénéficiaient en 2002 d'une exonération de $180 €$, contre $280 €$ par mois pour les entreprises à 35 heures. Le mon-

3. Le critère retenu pour classer ces entreprises est fonction de la proportion de salariés concernés par l'un de ces deux dispositifs. Si au moins 50 \% des salariés d'une entreprise multi-établissements bénéficient des allègements "Fillon-autres employeurs " on attribue ce type à l'ensemble des salariés de l'entreprise. 4. Le montant des exonérations par salarié bénéficiaire n'est pas calculé en utilisant les informations Acoss sur les salariés bénéficiaires (variable effrec). En effet, l'utilisation de cette variable conduit à estimer des niveaux d'allègements anormalement bas (par exemple inférieurs à $50 €$ pour les entreprises Fillon-Aubry 2 avant 2002). Ceci est lié au fait que les informations sur les effectifs dans les bordereaux récapitulatifs de cotisations sont de mauvaise qualité car mal renseignées. C'est pourquoi le nombre de salariés concernés par les exonérations est calculé à partir des données DADS.

Encadré 1

\section{LES DONNÉES}

Les fichiers de l'Acoss (bases Arome, Orme et Sequoia) permettent d'identifier différentes catégories d'établissements ayant bénéficié de dispositifs d'allègements de cotisations sociales sur la période 1999-2005. II s'agit principalement de la ristourne sur les bas salaires, des aides associées à la première loi Aubry sur la réduction du temps de travail et à la seconde loi Aubry sur le passage aux 35 heures (cf. annexe 1) et enfin des deux volets de la réforme Fillon de 2003, ceux touchant les établissements aux 35 heures et ceux affectant les autres établissements.

Ces fichiers sont appariés à la base DADS (Déclaration annuelle de données sociales) afin d'obtenir des informations sur l'évolution des effectifs, de la structure par tranche de salaires et par qualification de la maind'œuvre, de la répartition sectorielle, du type d'aides obtenues, du nombre de salariés concernés et du montant des exonérations de cotisations sociales dont bénéficie l'établissement.

Afin de disposer d'informations sur la situation économique des entreprises (valeur ajoutée, production, excédent brut d'exploitation,...), on effectue enfin un appariement avec le fichier Suse (Système unifié de statistiques d'entreprises).

Ces différents fichiers portent simultanément sur des données d'établissements (fichiers Sequoia, Orme, Arome et Dads) et d'entreprises (fichiers Suse). Pour harmoniser ces différentes sources, les données d'établissements sont agrégées en vue d'obtenir des informations au niveau de l'entreprise. Seules les entreprises pour lesquelles l'ensemble des établissements est présent simultanément dans les différents fichiers ont été retenues.

L'échantillon final résulte donc de l'appariement de trois sources administratives, les fichiers Suse,
DADS, Arome, Orme et Sequoia. L'échantillon cylindré obtenu porte sur plus de 92000 entreprises. Le champ retenu pour l'analyse est celui des entreprises de plus de cinq salariés (effectifs bruts renseignés au 31 décembre). Selon les études de la Dares, dans les entreprises de moins de cinq salariés, près d'un salarié sur deux est en contrat aidé (Belleville, SaintMartin, 2002), or ces contrats sont souvent assortis d'exonérations exclusives des allègements généraux. De surcroît, nous mobilisons la répartition des effectifs par tranche de Smic et dans les très petites entreprises ; cette information est difficile à obtenir ce qui justifie ce seuil de 5 salariés. On se limite par ailleurs à un échantillon cylindré d'entreprises présentes dans l'échantillon sur la période 2002 à 2005 et bénéficiant des allègements «Fillon-Aubry 2 » et “Fillon-autres employeurs" de manière continue sur la période 2003 à 2004 (bases Arome, Orme et Sequoia de l'Acoss).

Les entreprises bénéficiant simultanément des deux types d'aides, les holdings, les entreprises de services domestiques, les entreprises d'intérim ainsi que les entreprises publiques ont été supprimées. De même, les entreprises issues du secteur des hôtels-cafés-restaurants ont été écartées car elles ont fait l'objet d'une aide forfaitaire spécifique à ce secteur.

Afin de quantifier la hausse de la GMR supportée par les entreprises aux 35 heures, il est nécessaire d'identifier leur date de passage aux 35 heures. Cette information est obtenue à partir des données sur l'obtention des aides structurelles et incitatives versées aux entreprises sur la période 1999 à 2002 disponibles dans les fichiers de l'Acoss. II apparaît que la majorité des entreprises sont assujetties aux GMR 4 et 5, c'està-dire celles dont le montant a le moins augmenté sur la période 2002 à 2005 (cf. annexe 1). 
tant moyen d'exonération par salarié était quant à lui plus élevé pour les entreprises aux 39 heures que pour les entreprises à 35 heures (de l'ordre de $20 €$ ). En effet, avant 2003, les entreprises à 35 heures bénéficiaient d'allègements pour l'ensemble de leurs salariés, mais ces allégements étaient de faible ampleur (autour de 50 $€$ au titre de ceux dont la rémunération dépassait $1,7 \mathrm{Smic})$.

Un éclairage synthétique est donné par le taux d'exonération (TE) qui rapporte pour chaque entreprise le montant des exonérations au salaire brut. Entre 2002 et 2005 , ce taux a progressé de 2,5 points de pourcentage pour les entreprises aux 39 heures et a baissé de 1,5 point de pourcentage pour les entreprises à 35 heures, ce qui est conforme à ce que l'on attend compte tenu du profil des barèmes avant et après la réforme (cf. graphique I). La distribution de ces variations de taux selon la catégorie d'entreprises montre que la baisse du taux d'exonération concerne majoritairement les entreprises à 35 heures $(63 \%)$ et la hausse, les entreprises aux 39 heures (90\%) (cf. graphique II). Cette hausse est dans la majeure partie des cas assez accentuée : pour $58 \%$ des entreprises de cette catégorie, le taux d'exonération a augmenté de plus de 2 points de pourcentage. La baisse affectant les entreprises aux 35 heures est nettement moins accentuée : $33 \%$ seulement ont subi une

\section{Graphique II \\ Densité de l'évolution du taux d'exonération entre 2002 et 2005}

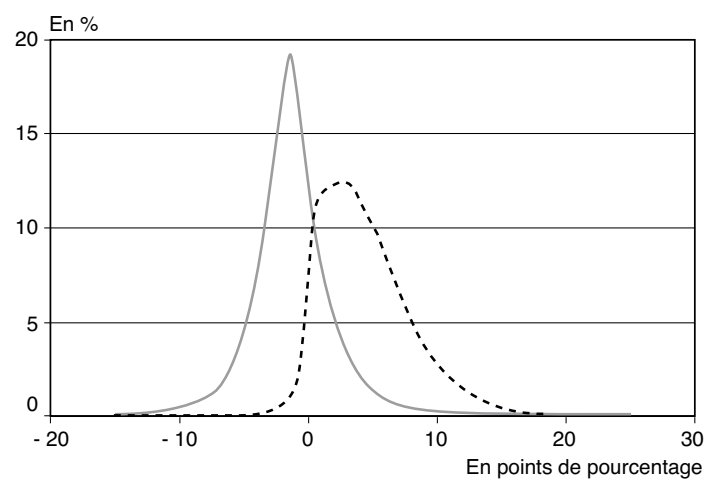

Écart de taux d'éxonération

- . - Entreprises à 39 heures _- Entreprises à 35 heures

Lecture : le graphique représente la distribution des entreprises selon la variation du taux d'exonération qu'elles ont connue entre 2002 et 2005, figurant sur l'axe horizontal. La surface sous chaque courbe est de $100 \%$. On constate que les entreprises aux 35 heures ont subi globalement une baisse de leur taux d'exonération alors que les entreprises à 39 heures ont plutôt expérimenté une hausse de leur taux d'exonération.

Champ : panel de 92939 entreprises de 5 salariés et plus sur les années 2002 à 2005 issues des secteurs d'activité privés et non agricoles.

Source : bases Arome, Orme et Sequoia (Acoss), DADS et Suse (Insee). baisse de ce taux d'au moins deux points de pourcentage.

\section{... et dans les secteurs des transports, du commerce et de la construction}

Le taux d'exonération a tendance à décroître avec la taille des entreprises du fait de la structure de leur main-d'œuvre (Acoss-Stat, 2005). Cette relation se vérifie dans l'échantillon utilisé dans cet article, notamment au premier semestre 2005. Néanmoins, cette corrélation n'est pas observée en 2002 , i.e avant la réforme étudiée ici, pour les entreprises à 39 heures.

Le taux d'exonération varie fortement selon le secteur d'activité. Par exemple, en 2005, le taux d'exonération dont bénéficient les entreprises des secteurs du commerce et du transport est près de deux fois supérieur à celui observé dans l'industrie ou les activités financières. Par ailleurs, c'est dans le secteur des transports que les entreprises ont le plus profité de la réforme de 2003 : puisque leur taux d'exonération a augmenté de trois points (et même de six points pour celles d'entre elles qui étaient restées aux 39 heures). En revanche, dans les secteurs des activités financières et immobilières et de l'industrie la situation est restée globalement stable, une nette amélioration pour les entreprises à 39 heures venant compenser la dégradation des entreprises à 35 heures.

La répartition sectorielle des entreprises passées aux 35 heures explique pour une part que la réforme de 2003 ait davantage profité aux entreprises du secteur des transports, du commerce et de la construction qu'au secteur de l'industrie et des activités financières et immobilières.

\section{Entre 2002 et 2005, le coût du travail a davantage augmenté dans les entreprises à moins de 35 heures}

La loi de 2003 a également joué sur le coût du travail : ce dernier a en moyenne pour l'ensemble des entreprises augmenté de 9,9\% entre 2002 et 2005.

Cette augmentation est plus marquée dans les entreprises à 35 heures que dans les autres $(12,2 \%$ contre $7,4 \%)$ (cf. graphique III). La progression de ce coût est supérieure ou égale à $10 \%$ dans plus de $46 \%$ des entreprises du premier groupe et dans seulement $38 \%$ du second. Inversement, il a baissé d'au moins $10 \%$ dans 
moins de $15 \%$ des entreprises du premier groupe et dans $22 \%$ du second. Les différences selon le secteur d'activité et la taille des entreprises sont aussi relativement importantes. Le coût du travail a augmenté près de deux fois moins dans l'industrie que dans les autres secteurs. Les augmentations les plus importantes ont été observées dans le secteur de la construction. Les entreprises de plus de 50 salariés ont connu une hausse de leur coût de l'ordre de $10,1 \%$ en moyenne contre $6,8 \%$ pour les autres.

\section{Déterminer l'évolution du coût du travail spécifiquement liée à la réforme de 2003}

Les évolutions du coût du travail observées entre 2002 et 2005 et mentionnées précédemment ne permettent pas de mesurer l'impact de la réforme de 2003 sur le comportement des entreprises. En effet, ces évolutions combinent deux éléments. Le premier est lié aux changements législatifs introduits par la loi de 2003. Le second provient des changements dans la structure des salaires et de la main-d'œuvre des entreprises qui sont en partie induits par cette réforme, ce qui est susceptible de générer un biais d'endogénéité de la variable de traitement.

\section{Graphique III \\ Densité de l'évolution du coût du travail par tête entre 2002 et 2005}

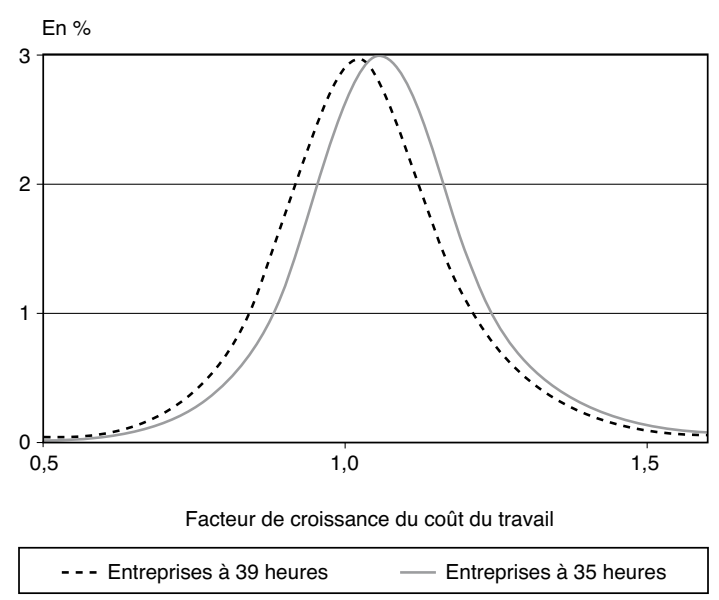

Lecture : le graphique représente la distribution des entreprises selon la progression du coût du travail qu'elles ont connue entre 2002 et 2005, le facteur de croissance du coût du travail figurant sur l'axe horizontal. La surface sous chaque courbe est de $100 \%$. Même s'il y a beaucoup de différences selon les entreprises, on constate globalement que les entreprises aux 35 heures ont subi une hausse de leur coût du travail plus forte que celle qui a été expérimentée par les entreprises à 39 heures.

Champ : panel de 92939 entreprises de 5 salariés et plus sur les années 2002 à 2005 issues des secteurs d'activité privés non agricoles.

Source : bases Arome, Orme et Sequoia (Acoss), DADS et Suse (Insee).
Afin de se prémunir contre ce biais, on s'inspire d'une méthode proposée dans un cas de figure similaire par Crépon et Desplatz (2001). Pour évaluer les effets des allègements de cotisations sociales sur les bas salaires, ces auteurs ont calculé pour chaque salarié un coût du travail virtuel en fonction du salaire observé de 1994 et des barèmes d'allègement de 1997. Cette démarche permet de tester l'impact de la réforme indépendamment des changements de rémunération observés entre 1994 et 1997. En effet, ces changements sont susceptibles d'influencer les variables d'intérêt et de biaiser ainsi l'estimation de l'effet du traitement. Pour éviter ce type de biais d'endogénéité et isoler l'effet spécifique de la réforme de 2003, on calcule un coût du travail qui ne tient compte que de la situation initiale et des changements exogènes (la situation des entreprises à l'égard des 35 heures, la structure des effectifs avant le choc, l'évolution des barèmes de cotisations sociales et la hausse des salaires minima).

\section{Les effets de la réforme sur l'évolution du coût du travail}

Pour calculer l'évolution du coût du travail induite spécifiquement par la réforme de 2003, seules les informations observées en 2002 (salaire moyen total et par tranche de Smic, composition de la main-d'œuvre, situation à l'égard des 35 heures) et les changements affectant le coût du travail liés à cette réforme (changement de barèmes et augmentation du Smic/ GMR) sont mis à contribution.

Dans un premier temps, on calcule un taux d'exonération virtuel (TEV) lié spécifiquement à la réforme de 2003. Le taux d'exonération utilisé plus haut rapportait, pour chaque entreprise, le total des exonérations réelles (c'est-à-dire dont l'entreprise a effectivement bénéficié) et le salaire brut. Le taux d'exonération virtuel (TEV) repose sur la même définition mais les allègements sont cette fois des allègements virtuels, calculés pour chaque entreprise en fonction des cotisations sociales employeur, des barèmes d'allègements légaux, de l'évolution du Smic et de la GMR et de la distribution de la maind'œuvre par niveau de salaire (cf. annexe 3 ).

Cette opération utilise plusieurs types d'information. Les données DADS fournissent la répartition des salariés par tranche de salaire (huit tranches ont été retenues), les données Acoss permettent d'observer pour chaque trimestre le montant des allègements obtenus et des cotisations sociales employeurs versées. Elles rendent 
également possible le contrôle de qualité du TEV. Pour le second semestre de l'année 2002, il est en effet possible d'analyser l'écart entre le

\section{Graphique IV}

Densité de l'évolution du coût du travail induite par les mesures Fillon entre 2002 et 2005

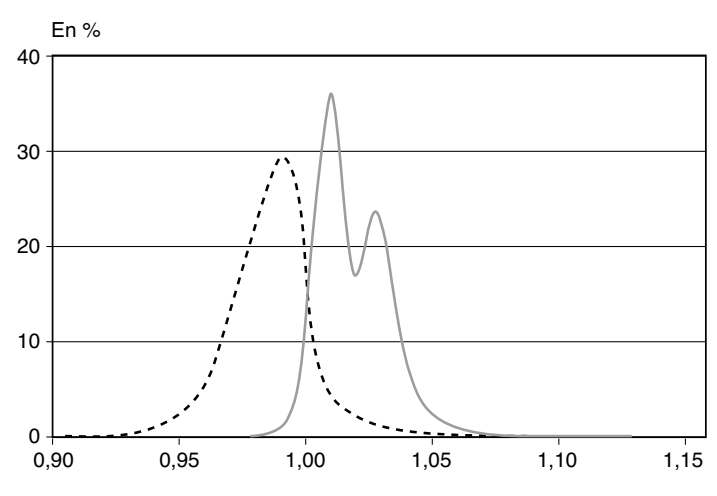

Facteur de croissance du coût du travail

- - - Entreprises à 39 heures — Entreprises à 35 heures

Lecture : le graphique représente la distribution des entreprises selon la progression du coût du travail qu'elles ont connue entre 2002 et 2005, en se focalisant sur la variation du coût du travail induite directement par la réforme de janvier 2003. La surface sous chaque courbe est de $100 \%$. On constate globalement que la réforme de janvier 2003 a conduit à une hausse du coût du travail dans les entreprises aux 35 heures alors qu'elle a plutôt diminué le coût du travail des entreprises à 39 heures.

Champ : panel de 92939 entreprises de cinq salariés et plus sur les années 2002 à 2005 issues des secteurs d'activité privés non agricoles.

Source : bases Arome, Orme et Sequoia (Acoss), DADS et Suse (Insee).
TEV et TE réellement observé (5), ce qui conduit à émettre des réserves sur les données fournies par les DADS et par la base des accords RTT de la Dares.

Ce TEV donne une mesure de l'impact spécifique de la réforme de 2003 sur le coût du travail. Globalement, cette réforme a eu un impact très faible sur le coût du travail avec une augmentation de l'ordre de $+0,4 \%$. Cependant, il existe des différences importantes entre les entreprises à 39 heures et les autres, de l'ordre de 2,5 points de pourcentage (cf. graphique IV). Le coût du travail a eu tendance à baisser dans plus de $80 \%$ des cas dans les entreprises aux 39 heures (- $1 \%$ en moyenne); en revanche, il a augmenté (de $+1,6 \%$ en moyenne) dans les entreprises à 35 heures.

L'évolution observée du coût du travail (cf. graphique III) et celle qui a été induite par la réforme de 2003 (cf. graphique IV) sont très différentes. Pour bien le visualiser, nous représentons les distributions sur un même plan en distinguant les entreprises à 35 heures et celles aux 39 heures (cf. graphique V). L'évolution du coût du travail a été en réalité beaucoup plus dispersée selon les entreprises que celle qui a

5. En 2001, Crépon et Desplatz n'avaient pu se livrer à un tel contrôle, faute de données sur les cotisations réellement versées.

\section{Graphique V}

Densités de évolutions effectives du coût du travail et des évolutions induites par les mesures Fillon entre 2002 et 2005

\section{A - Entreprises aux 39 heures}

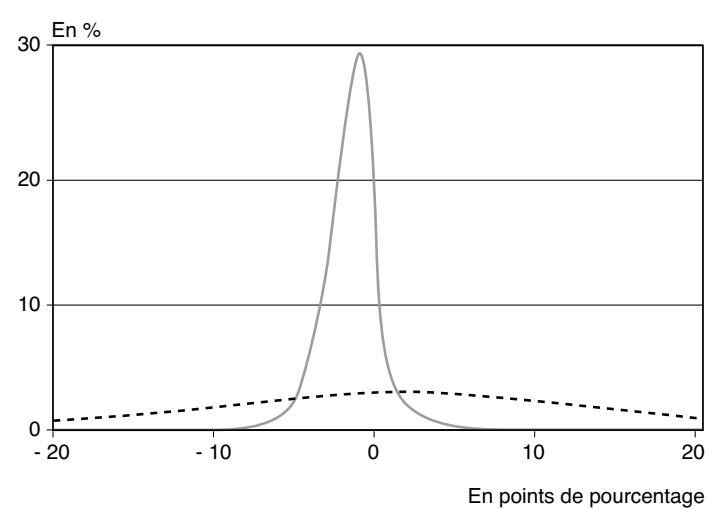

Taux de croissance du coût du travail

À structure constante

\section{B - Entreprises aux 35 heures}

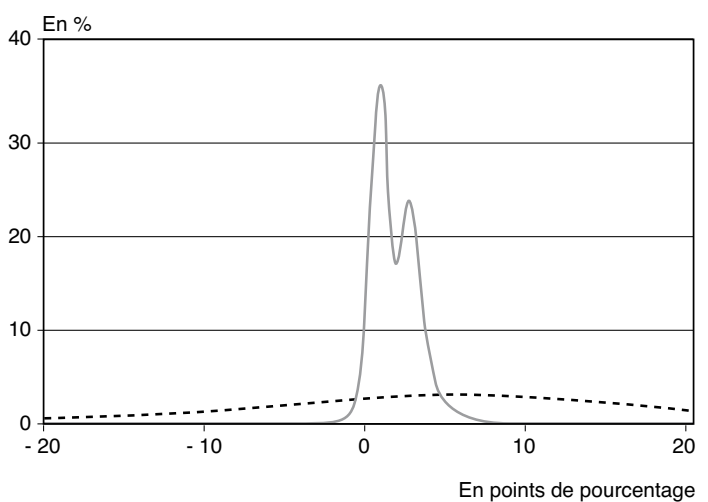

Taux de croissance du coût du travail

- - - Observe
- À structure constante

Lecture : afin de mieux visualiser l'intérêt de la correction que nous avons effectué pour mesurer l'effet de la réforme de 2003 sur le coût du travail, nous superposons sur ce graphique la progression du coût du travail effective et celle qui est associée aux seuls effets de la réforme (à structure constante). Les graphiques représentent la distribution des entreprises selon la progression du coût du travail qu'elles ont connue entre 2002 et 2005, en comparant la variation du coût du travail effective et celle induite directement par la réforme de janvier 2003. La surface sous chaque courbe est de $100 \%$. On constate que si les entreprises ont expérimenté des évolutions du coût du travail très différenciées entre 2002 et 2005, l'effet spécifique de la réforme tel que nous l'avons estimé est beaucoup plus concentré. Champ : panel de 92939 entreprises de cinq salariés et plus sur les années 2002 à 2005 issues des secteurs d'activité privés non agricoles. Source : bases Arome, Orme et Sequoia (Acoss), DADS et Suse (Insee). 
été induite uniquement par les hausses successives des salaires minima et des changements de barèmes d'exonération de la réforme de 2003 . Entre 2002 et 2005, les entreprises ont pris de multiples décisions d'embauche et de licenciement et de hausses de salaire. Certaines de ces décisions ont été sans doute motivées par les effets de la réforme alors que d'autres en étaient indépendantes. Elles ont entraîné une modification de la structure des entreprises en matière d'emploi et de salaires. L'évolution du coût du travail est donc beaucoup plus dispersée lorsque l'on ne l'observe pas à structure constante. Cela justifie de distinguer l'évolution effective du coût du travail et celle qui a été induite par la réforme.

\section{La loi de 2003 bénéficie essentiellement aux petites entreprises restées à 39 heures avec un personnel mal rémunéré}

Si l'on se réfère cette fois au taux de croissance du coût du travail directement lié à la loi de 2003, les entreprises qui ont enregistré une diminution du coût du travail (variation comprise entre $-3 \%$ et $0 \%$ ) sont dans plus de $90 \%$ des cas des entreprises restées aux 39 heures. À l'inverse, dans plus de $75 \%$ des cas, les entreprises dans lesquelles la variation du coût du travail directement imputable à cette loi est positive ( $+0,5 \%$ et plus) sont à 35 heures. Parmi ce dernier groupe d'entreprises, celles qui ont subi la plus forte hausse de leur coût ( $+2 \%$ et plus) sont celles qui bénéficiaient jusque-là des aides
Aubry 1 (dans plus de $90 \%$ des cas). Ces aides incitatives d'une durée maximale de cinq ans arrivaient à échéance en 2004 (pour les entreprises de plus de 20 salariés).

Ainsi qu'il était prévisible, ces baisses de coût $\mathrm{du}$ travail sont particulièrement importantes dans des entreprises restées aux 39 heures et employant une forte proportion de salariés rémunérés entre 1,2 et $1,7 \mathrm{Smic}(44,4 \%$ de celles pour lesquelles la diminution du coût du travail se situe entre $1 \%$ et $2 \%$ ). Les entreprises concernées proviennent moins fréquemment des secteurs de l'industrie ( $27 \%$ à $42 \%$ des cas) et sont plus souvent constituées d'entreprises de petite taille. En revanche, les entreprises qui ont connu une augmentation du coût du travail sont plus particulièrement celles passées à 35 heures. Il s'agit le plus souvent de grandes entreprises (dans au moins $50 \%$ des cas, elles comptent 200 salariés et plus) et une large part de leurs travailleurs perçoit une rémunération élevée (de $44,6 \%$ à 56,8 \% des entreprises ayant connu une hausse du coût du travail comprise entre 1 et $2 \%$ ) (cf. tableaux 1 à 3 ).

\section{Les baisses de coût du travail coïncident avec des hausses de l'emploi et des salaires}

La comparaison des entreprises passées à 35 heures à celles restées à 39 heures quant à l'évolution des salaires et de l'emploi au cours de la période encadrant la réforme de 2003 (années 2002 à 2005) révèle une certaine diversité de situations. Cette diversité est cohérente avec les

\section{Tableau 1}

Répartition des entreprises selon qu'elles sont ou non restées à 39 heures en fonction de l'évolution du coût du travail directement induit par la loi de 2003

\begin{tabular}{|c|c|c|c|c|c|c|c|c|c|}
\hline & \multicolumn{9}{|c|}{ Taux d'évolution du coût du travail directement induit par les lois Fillon } \\
\hline & $\begin{array}{l}\text { Inférieur } \\
\text { à - } 3\end{array}$ & $\begin{array}{c}\text { Compris } \\
\text { entre - } 3 \\
\text { et - } 2\end{array}$ & $\begin{array}{l}\text { Compris } \\
\text { entre - } 2 \\
\text { et - } 1\end{array}$ & $\begin{array}{l}\text { Compris } \\
\text { entre - } 1 \\
\text { et - } 0,03\end{array}$ & $\begin{array}{c}\text { Compris } \\
\text { entre } \\
-0,03 \\
\text { et }+0,05\end{array}$ & $\begin{array}{c}\text { Compris } \\
\text { entre } \\
0,5 \text { et } 1\end{array}$ & $\begin{array}{c}\text { Compris } \\
\text { entre } \\
1 \text { et } 2\end{array}$ & $\begin{array}{l}\text { Compris } \\
\text { entre } \\
2 \text { et } 3\end{array}$ & $\begin{array}{l}\text { Supérieur } \\
\text { à } 3\end{array}$ \\
\hline $\begin{array}{l}\text { Entreprises } \\
\text { à } 39 \text { heures }\end{array}$ & 100,0 & 100,0 & 99,8 & 93,1 & 33,8 & 24,7 & 10,2 & 6,9 & 4,9 \\
\hline $\begin{array}{l}\text { Entreprises } \\
\text { à } 35 \text { heures }\end{array}$ & 0,0 & 0,0 & 0,2 & 6,9 & 66,2 & 75,3 & 89,9 & 93,1 & 95,1 \\
\hline $\begin{array}{l}\text { Dont: } \\
\text { Entreprises } \\
\text { bénéficiant des } \\
\text { aides Aubry } 1\end{array}$ & 0,0 & 0,0 & 0,0 & 0,3 & 3,9 & 4,8 & 8,4 & 85,4 & 93,5 \\
\hline $\begin{array}{l}\text { Nombre } \\
\text { d'observations }\end{array}$ & 9100 & 10790 & 16124 & 17616 & 6744 & 6994 & 9884 & 7873 & 7873 \\
\hline
\end{tabular}

Lecture : $24,7 \%$ des entreprises pour lesquelles la variation du coût du travail est comprise entre $0,5 \%$ et $1 \%$ lors de la mise en place de la réforme Fillon (2003) sont des entreprises restées aux 39 heures.

Champ : panel de 92939 entreprises de cinq salariés et plus au cours des années 2002 à 2005 appartenant aux secteurs d'activité privés non agricoles.

Source : bases Arome, Orme et Sequoia (Acoss), DADS et Suse (Insee). 
Tableau 2

Caractéristiques des entreprises selon l'évolution du coût du travail directement induit par la loi de 2003 - entreprises à 39 heures

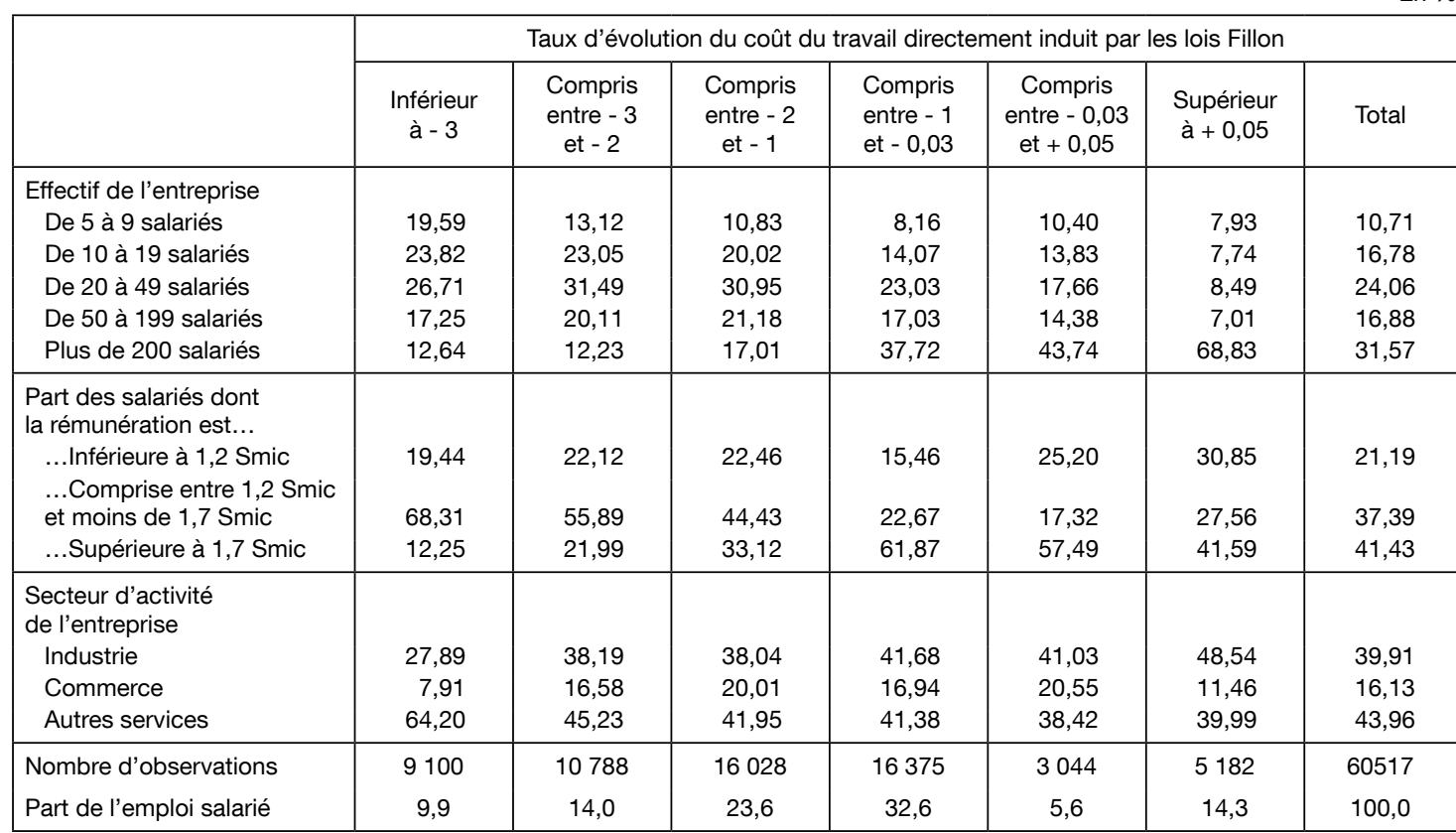

Lecture : en pourcentages de l'effectif salarié total des entreprises à 39 heures. 26,71\% des salariés des entreprises restées aux 39 heures dans lesquelles le coût du travail a diminué de plus de $3 \%$ en conséquence directe de la réforme Fillon (2003) travaillent dans des entreprises comptant entre 20 et 50 salariés; les entreprises aux 39 heures dans lesquelles la hausse du coût du travail (directement induite par les lois Fillon) a été supérieure à 0,05\% comportent 30,85\% de salariés rémunérés à un salaire inférieur à 1,2 Smic.

Champ : panel de 92939 entreprises de cinq salariés et plus au cours des années 2002 à 2005 appartenant aux secteurs d'activité privés non agricoles.

Source : bases Arome, Orme et Sequoia (Acoss), DADS et Suse (Insee).

Tableau 3

Caractéristiques des entreprises selon l'évolution du coût du travail directement induit par la loi de 2003 - entreprises à 35 heures

\begin{tabular}{|c|c|c|c|c|c|c|}
\hline & \multicolumn{6}{|c|}{ Taux d'évolution du coût du travail directement induit par les lois Fillon } \\
\hline & $\begin{array}{l}\text { Inférieur } \\
\text { à } 0,5\end{array}$ & $\begin{array}{c}\text { Compris } \\
\text { entre } 0,5 \text { et } 1\end{array}$ & $\begin{array}{l}\text { Compris } \\
\text { entre } 1 \text { et } 2\end{array}$ & $\begin{array}{l}\text { Compris } \\
\text { entre } 2 \text { et } 3\end{array}$ & $\begin{array}{l}\text { Supérieur } \\
\text { à } 3\end{array}$ & Total \\
\hline $\begin{array}{l}\text { Effectif de l'entreprise } \\
\text { De } 5 \text { à } 9 \text { salariés } \\
\text { De } 10 \text { à } 19 \text { salariés } \\
\text { De } 20 \text { à } 49 \text { salariés } \\
\text { De } 50 \text { à } 199 \text { salariés } \\
\text { Plus de } 200 \text { salariés }\end{array}$ & $\begin{array}{r}4,62 \\
7,47 \\
23,45 \\
24,60 \\
39,86\end{array}$ & $\begin{array}{r}2,73 \\
4,83 \\
18,15 \\
25,25 \\
49,04\end{array}$ & $\begin{array}{r}1,87 \\
3,18 \\
9,50 \\
15,23 \\
70,22\end{array}$ & $\begin{array}{r}5,39 \\
7,62 \\
15,78 \\
23,90 \\
47,31\end{array}$ & $\begin{array}{r}5,98 \\
7,07 \\
17,00 \\
20,01 \\
49,94\end{array}$ & $\begin{array}{r}3,53 \\
5,26 \\
14,72 \\
20,12 \\
56,36\end{array}$ \\
\hline $\begin{array}{l}\text { Part des salariés dont } \\
\text { la rémunération est... } \\
\ldots \text {.. Inférieure à } 1,2 \text { Smic } \\
\ldots \text {..Comprise entre } 1,2 \text { Smic et } \\
\text { moins de } 1,7 \text { Smic } \\
\text {...Supérieure à } 1,7 \text { Smic }\end{array}$ & $\begin{array}{l}23,50 \\
49,35 \\
27,15\end{array}$ & $\begin{array}{l}18,03 \\
46,47 \\
35,49\end{array}$ & $\begin{array}{l}10,21 \\
32,99 \\
56,79\end{array}$ & $\begin{array}{l}14,69 \\
40,66 \\
44,65\end{array}$ & $\begin{array}{l}30,39 \\
45,69 \\
23,91\end{array}$ & $\begin{array}{l}16,89 \\
40,33 \\
42,78\end{array}$ \\
\hline $\begin{array}{l}\text { Secteur d'activité de l'entreprise } \\
\text { Industrie } \\
\text { Commerce } \\
\text { Autres services }\end{array}$ & $\begin{array}{r}44,13 \\
3,54 \\
52,33\end{array}$ & $\begin{array}{r}50,89 \\
3,03 \\
46,08\end{array}$ & $\begin{array}{r}60,19 \\
9,48 \\
30,34\end{array}$ & $\begin{array}{l}43,73 \\
10,56 \\
45,71\end{array}$ & $\begin{array}{l}32,95 \\
13,06 \\
53,99\end{array}$ & $\begin{array}{r}49,91 \\
8,45 \\
41,64\end{array}$ \\
\hline $\begin{array}{l}\text { Entreprises bénéficiant des aides } \\
\text { incitatives Aubry } 1\end{array}$ & 4,4 & 4,1 & 8,8 & 88,7 & 96,9 & 34,2 \\
\hline $\begin{array}{l}\text { Nombre d'observations } \\
\text { Part de l'emploi salarié }\end{array}$ & $\begin{array}{l}5039 \\
11,28\end{array}$ & $\begin{array}{l}5427 \\
16,86\end{array}$ & $\begin{array}{l}8074 \\
40,11\end{array}$ & $\begin{array}{l}7029 \\
16,22\end{array}$ & $\begin{array}{l}6853 \\
15,53\end{array}$ & $\begin{array}{c}32422 \\
100\end{array}$ \\
\hline
\end{tabular}

Lecture : en pourcentages de l'effectif salarié total des entreprises passées aux 35 heures. Les données sont des moyennes sur la période 2002-2005. Se reporter au tableau 2.

Champ : panel de 92939 entreprises de cinq salariés et plus au cours des années 2002 à 2005 appartenant aux secteurs d'activité privés non agricoles.

Source : bases Arome, Orme et Sequoia (Acoss), DADS et Suse (Insee). 
constats précédents sur l'évolution du coût du travail dans les deux types d'entreprises.

Dans les entreprises à 39 heures, la hausse de l'emploi a été d'autant plus forte entre 2002 et 2005 que la baisse du coût du travail a été prononcée (cf. tableau 4), et cela, en termes d'effectifs occupés aussi bien qu'en équivalents temps complet. La relation apparente entre coût du travail et emploi est moins nette dans le cas des entreprises restées à 35 heures.

Sur la même période, quel que soit le type d'entreprise, l'accroissement des salaires est d'autant plus élevé que la hausse du coût induite par la réforme est faible. Par ailleurs, les salariés des entreprises aux 39 heures ont vu leur rémunération augmenter davantage que ceux des entreprises à 35 heures $(6,06 \%$ contre $5,27 \%)$. Notons que cet indicateur reflète simultanément l'évolution de trois variables : le temps de travail des salariés à taux plein, la part des salariés à temps partiel et le recours aux emplois temporaires.

Au total, par rapport aux autres entreprises, les entreprises aux 39 heures qui ont plutôt expérimenté une baisse de coût du travail avec la mise en place des dispositifs Fillon, voient l'emploi et les rémunérations de leurs salariés progresser plus vite que les entreprises à 35 heures entre 2002 et 2005.
Néanmoins, ces corrélations ne reflètent pas nécessairement un effet de la loi de 2003. Nous avons vu que les deux groupes d'entreprises, celles à 35 heures et celles restées aux 39 heures, présentaient des caractéristiques particulières en termes de composition de la main d'œuvre employée, de secteur d'activité ou encore d'indicateurs comptables et financiers (cf. annexe 2). Par ailleurs, les travaux de Bunel (2005) et Gilles (2006) soulignent que ces entreprises se singularisent également en termes de technologie, d'organisation et d'horaires de travail. Il est nécessaire à ce stade de réaliser une analyse économétrique afin de contrôler d'éventuels effets de composition.

\section{Mesurer l'impact de l'évolution du coût du travail « toutes choses égales par ailleurs »)}

La variation du coût du travail au niveau de l'entreprise est sujette à différentes erreurs de mesure liées à l'usage de sources administratives et à des problèmes de déclarations ou d'enregistrement. En vue de limiter l'influence de ces erreurs, nous retenons comme variable de traitement la position des entreprises par rapport à la distribution de cette variation, en distinguant différentes classes de valeurs.

\section{Tableau 4 \\ Évolution du coût du travail directement liée à la réforme de 2003 et évolution des niveaux d'emploi et de salaire entre 2002 et 2005}

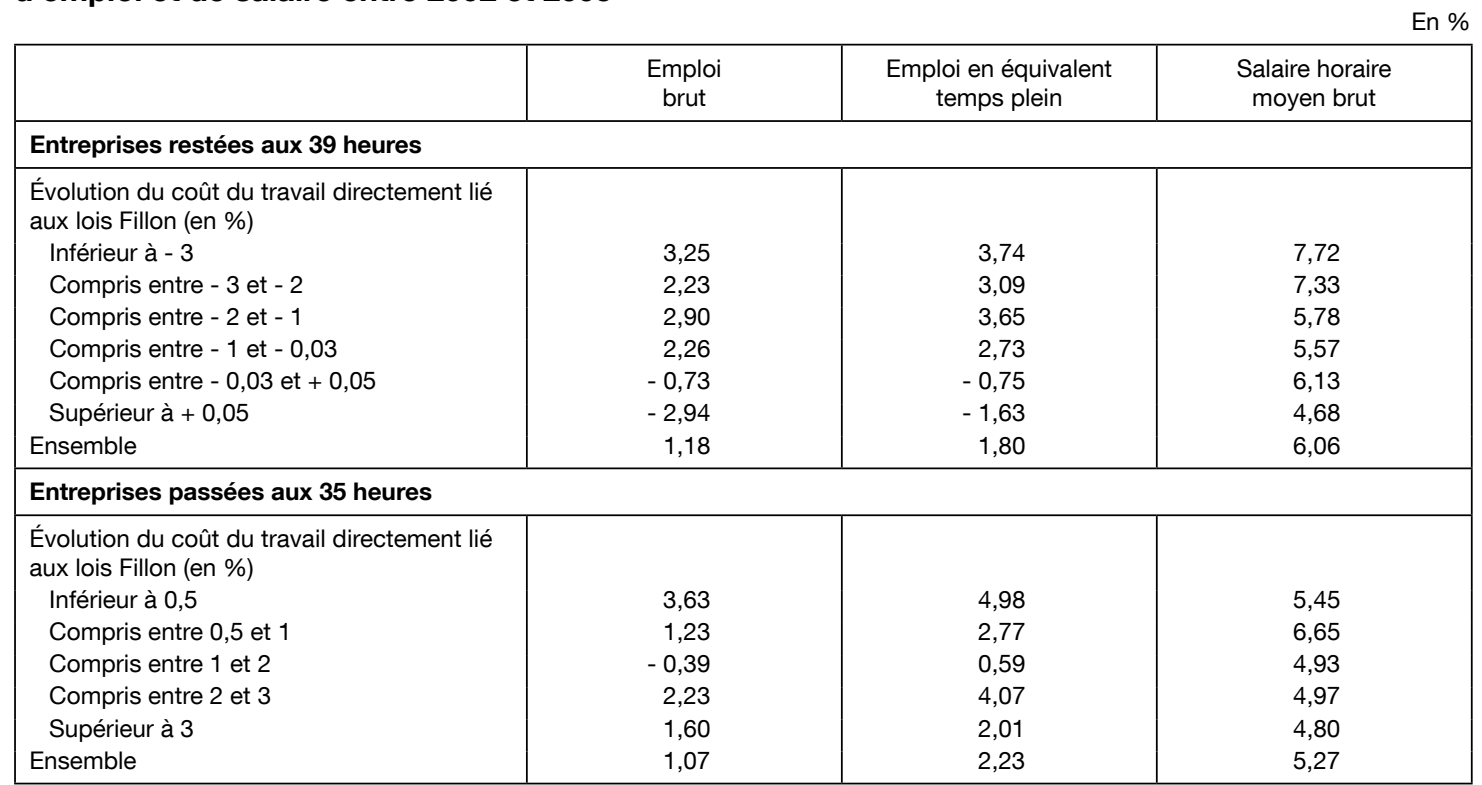

Lecture : dans les entreprises restées aux 39 heures et ayant enregistré une variation du coût du travail comprise entre - $3 \%$ et - $2 \%$ entre 2002 et 2005, le niveau de l'emploi brut a augmenté de 2,23\%, l'emploi en équivalent temps plein de 3,09\% et le salaire horaire moyen brut s'est accru de $7,33 \%$.

Champ : panel de 92939 entreprises de cinq salariés et plus au cours des années 2002 à 2005 appartenant aux secteurs d'activité privés non agricoles.

Source : bases Arome, Orme et Sequoia (Acoss), DADS et Suse (Insee). 
Afin de tester « toutes choses égales par ailleurs ", l'impact d'une variation relative du coût du travail sur l'évolution de l'emploi et des salaires (considérées comme variables d'intérêt) pour chaque sous-population, on a recours à trois méthodes économétriques : la méthode des moindres carrés ordinaires $(\mathrm{MCO})$; la méthode de la régression quantile $(\mathrm{QR})$; la méthode $\mathrm{du}$ score de propension (PSM). Combiner plusieurs techniques économétriques, paramétriques puis semi-paramétriques, permet de mettre en évidence des effets indépendants du choix de telle ou telle technique. L'estimation de référence s'appuie sur la méthode des moindres carrés ordinaires (MCO) qui permet d'évaluer l'effet moyen du traitement en s'appuyant sur une hypothèse d'exogénéité. Au moyen de régressions quantiles, on teste ensuite l'impact du traitement sur l'ensemble de la distribution des variables d'intérêt. Pour relâcher l'hypothèse de relation linéaire entre les variables expliquées et les variables explicatives ainsi que celle de normalité des résidus, des estimations sont enfin réalisées en utilisant les méthodes d'appariement sélectif sur le score de propension (cf. encadré 2).

\section{Résultats des estimations}

Pour chacun des groupes traités, les estimations économétriques fournissent l'ampleur du supplément de hausse (ou de baisse) de l'emploi à partir du groupe de contrôle correspondant (cf. encadré 2 et annexes 4 et 5). À partir de ce différentiel de création d'emplois et de la variation moyenne du coût du travail entre le groupe traité considéré et le groupe de contrôle, on calcule une élasticité propre à chaque groupe d'entreprises (cf. tableaux 5 à 8 ).

Ces élasticités de l'emploi et des salaires au coût du travail sont déterminées pour les entreprises passées aux 35 heures et pour celles restées aux 39 heures. Elles permettent de déterminer l'effet (mesuré en pourcentage) d'une hausse de $1 \%$ du coût du travail sur l'emploi (mesuré en effectif ou en équivalent temps complet) et sur les salaires. Elles sont souvent significatives et ont toujours le signe attendu. Globalement, une hausse du coût du travail de $1 \%$ réduit l'emploi d'environ $0,25 \%$.

Encadré 2

\section{MÉTHODES ÉCONOMÉTRIQUES}

La méthode des moindres carrés ordinaires estime de manière convergente et sans biais l'effet moyen du traitement si ce dernier est exogène. Le modèle s'écrit sous la forme suivante:

$$
\Delta \ln Y_{i}=X_{i} \beta+T_{i} \gamma+u_{i}
$$

où $\Delta \ln Y_{i}$ représente le taux de croissance de la variable expliquée (l'emploi, le coût du travail et le salaire horaire), $X_{i}$ un vecteur de variable explicative, $T_{i}$ le traitement et $u_{i}$ un terme d'erreur.

En complément des $\mathrm{MCO}$, la méthode de la régression quantile proposée initialement par Koenker et Bassett (1978) permet de mesurer l'effet de variables expliquées sur la variable estimée en différents points de sa distribution (et non pas uniquement à la moyenne comme pour le modèle linéaire estimé par les moindres carrées ordinaires). Cette méthode largement utilisée aujourd'hui (Koenker et Hallock, 2001) s'appuie sur le programme de minimisation suivant :

$$
\begin{aligned}
& \min \left(\sum_{i: \Delta \ln Y_{i} \geq X_{i} \beta_{\theta}+T_{i} \gamma_{\theta}} \theta\left|\Delta \ln Y_{i}-X_{i} \beta_{\theta}-T_{i} \gamma_{\theta}\right|\right. \\
& \left.\quad+\sum_{i: \Delta \ln Y<<_{i} X_{i} \beta_{\theta}+T_{i} \gamma_{\theta}}(1-\theta)\left|\Delta \ln Y_{i}-X_{i} \beta_{\theta}-T_{i} \gamma_{\theta}\right|\right)
\end{aligned}
$$

où $\theta$ représente le quantile retenu. Les paramètres estimés $\beta_{\theta}$ et $\gamma_{\theta}$ peuvent varier selon le quantile considéré. Dans notre cas, cette approche a pour attrait principal de nous permettre de contrôler l'homogénéité de l'impact d'une variation du coût du travail générée par les mesures Fillon (2003) sur l'ensemble de la distribution des variables d'intérêt.

Enfin, pour relâcher l'hypothèse de normalité et celle de linéarité entre la variable expliquée et les variables explicatives imposées par les deux méthodes précédentes, on a recours à la méthode semi-paramétrique du score de propension (PSM). Néanmoins, pour chaque entreprise, seule la situation correspondant à l'évolution du coût du travail effectivement obtenue est observable. La PSM permet d'étudier la situation qui aurait résulté d'une évolution alternative du coût du travail. Pour cela, on fait appel à la méthode de l'estimateur à noyau développée par Heckman, Ichimura et Todd (1998).

Cet estimateur repose sur la construction, pour chaque entreprise traitée (i.e. ayant par exemple bénéficié d'un plus fort accroissement des exonérations), d'une entreprise contrefactuelle consistant en une moyenne pondérée de l'ensemble des entreprises du groupe de contrôle (ou de référence). La pondération retenue est déterminée en fonction de la distance entre le score obtenu par une entreprise (i.e. la probabilité que l'entreprise ait bénéficié de plus d'exonérations condition- 
Ces résultats sont peu sensibles à la technique d'estimation. La méthode du score de propension (PSM) donne des résultats qualitativement comparables à l'approche paramétrique (MCO). L'estimation des scores est fournie en annexe 6. Les résultats de la régression quantile $(\mathrm{QR})$, quant à eux, laissent supposer que l'estimation des élasticités obtenues par les moindres carrés ordinaires est plus élevée dans la partie inférieure de la distribution pour les entreprises à 39 heures.

\section{Entreprises à 35 heures : la hausse du coût du travail aurait eu un effet négatif sur l'emploi et positif sur les salaires}

Dans le cas des entreprises passées à 35 heures, les élasticités de l'emploi au coût du travail sont plus souvent significatives lorsque l'emploi est mesuré en équivalent temps complet (cf. tableaux 5 et 6 ). En outre, puisque les entreprises à 35 heures ont majoritairement expéri-

\section{Encadré 2 (suite)}

nellement à ses caractéristiques) et celui de chacune des entreprises du groupe de contrôle.

Dans notre cas, toutes les entreprises ont été affectées par la mise en place de la réforme de 2003. Cependant, tant les entreprises aux 35 heures que les autres se distinguent entre elles par le fait qu'elles ont expérimenté des variations différentes du coût du travail. Nous avons donc construit des classes de valeurs de la variation du coût du travail dans les différentes entreprises. Pour les entreprises aux 35 heures, qui ont expérimenté plus fréquemment une hausse du coût du travail, ces classes sont les suivantes : moins de $0,5 \%$ d'augmentation; de 0,5 à $1 \%$; de $1 \%$ à $2 \%$; entre 2 et $3 \%$; et plus de $3 \%$. A l'inverse, pour les entreprises aux 39 heures, qui ont expérimenté plus fréquemment une baisse du coût du travail, ces classes sont les suivantes : un accroissement de plus de 0,05\% ; une variation comprise entre $-0,03$ et $+0,05 \%$; une variation de -1 à $-0,03 \%$; de -2 à $-1 \%$; de -3 à $-2 \%$ et une baisse de plus de $3 \%$.

Sur la base de ces classes de variation du coût du travail, on a choisi un groupe de contrôle pour chacun des deux types d'entreprises : l'ensemble des entreprises aux 35 heures ayant expérimenté une hausse de plus de $3 \%$ du coût du travail ; l'ensemble des entreprises aux 39 heures dans lesquelles la baisse du coût du travail a été supérieure à $3 \%$. Pour ces deux catégories d'entreprises, les groupes traités (le traitement revêt par conséquent ici plusieurs formes, chacun correspondant à l'une des classes restantes) sont constitués des entreprises appartenant aux classes restantes.

Pour les entreprises aux 35 heures, nous avons estimé la variation de l'emploi dans les entreprises où le coût du travail a augmenté de moins de $3 \%$ à la suite de la réforme Fillon (par exemple variation comprise entre $0,5 \%$ à $1 \%$ ou entre $1 \%$ et $2 \%$ ) par rapport à la situation dans laquelle elles auraient subi un accroissement du coût du travail supérieur à $3 \%$ (groupe de contrôle). À l'inverse, pour les entreprises restées aux 39 heures et ayant bénéficié plus fréquemment d'une baisse du coût du travail, nous avons estimé la variation de l'emploi qu'ont expérimentée ces entreprises lorsque leur coût du travail a baissé de moins de $3 \%$ (par exemple : variation comprise entre $-1 \%$ et $-0,03 \%$ ou entre $-2 \%$ et $-1 \%$ lors de la mise en place de la mesure, par rapport à la situation dans laquelle elles auraient bénéficié d'une baisse du coût du travail supérieure à 3 \% (groupe de contrôle pour ce second groupe d'entreprises).
Initialement l'estimateur de Heckman, Ichimura et Todd (1998) porte sur l'effet d'un traitement dichotomique. II est toutefois nécessaire dans notre cas de le généraliser au cas d'un traitement multiple ce qui est techniquement possible (Brodaty et alii, 2000 ; Lechner, 1999 ; Janod et Saint-Martin, 2004).

Notons $C_{T=k}^{k l}$ l'effet causal recherché qui correspond à l'écart d'un indicateur de performance $Y$ entre la situation où l'entreprise a bénéficié d'une variation $k$ du taux d'exonération apparent par rapport à une variation / avec $k>I$.

$$
C_{T=k}^{k l} \equiv E\left(\Delta \ln Y_{i k} \mid T_{i}=k\right)-E\left(\Delta \ln Y_{i l} \mid T_{i}=k\right)
$$

Comme pour tous les modèles d'appariement, la difficulté réside dans le fait que $E\left(Y_{i l} \mid T_{i}=k\right)$ n'est pas observable. On la contourne en estimant cet effet causal au moyen de l'estimateur suivant :

$$
\begin{aligned}
\widehat{C}_{T=k}^{k l} \equiv & \frac{1}{N_{k}} \sum_{i \in T_{k}} \\
& {\left[\Delta \ln Y_{i}-\sum_{j \in T_{k}} \frac{K\left[\left(\hat{p}_{i}^{k l}-\hat{p}_{j}^{k l}\right) / h_{j}\right]}{\sum_{j \in T_{k}} K\left[\left(\hat{p}_{i}^{k l}-\hat{p}_{j}^{k l}\right) / h_{j}\right]} \Delta \ln Y_{j}\right] }
\end{aligned}
$$

$N_{k}$ correspond au nombre d'entreprises ayant bénéficié d'une variation $k$ du coût du travail, et $T_{k}$ est l'ensemble comprenant ces entreprises $K$ [.] désigne une fonction noyau et $h_{j}$ un paramètre de lissage, fixé à 0,06 . Les probabilités $\hat{p}_{i}^{k l}$ sont déduites à partir des probabilités estimées issues d'un modèle probit ordonné qui nous permet de calculer $\operatorname{Pr}\left(T=k \mid X_{i}\right)$ et $\operatorname{Pr}\left(T=l \mid X_{i}\right)$. On a en effet :

$$
\hat{p}_{i}^{k l}=\frac{\operatorname{Pr}\left(T=k \mid X_{i}\right)}{\operatorname{Pr}\left(T=k \mid X_{i}\right)+\operatorname{Pr}\left(T=I \mid X_{i}\right)}
$$

Ces estimateurs sont calculés sur le support commun, c'est-à-dire sur la partie commune des deux densités des scores de propension des entreprises traitées et du groupe de contrôle (de référence). Les distributions de densité sont présentées en annexe 8. Enfin, pour obtenir les écarts-types asymptotiques, la méthode du bootstrap est mise en œuvre en effectuant 50 simulations consécutives. 
menté une augmentation du coût du travail avec la mise en place de la loi de 2003, la sensibilité négative de l'emploi en équivalent temps plein au choc $(-0,28)$ indique que le volume d'heures travaillées a diminué du fait, entre autres, d'un recours accru aux emplois temporaires courts et

Tableau 5

Estimation de l'élasticité des variables d'intérêt à l'évolution du coût du travail pour les entreprises à 35 heures

\begin{tabular}{|c|c|c|c|c|}
\hline & \multirow[t]{2}{*}{$\mathrm{MCO}$} & \multicolumn{3}{|c|}{ Régressions quantile } \\
\hline & & $25 \%$ & $50 \%$ & $75 \%$ \\
\hline Emploi brut & $\begin{array}{c}-0,0585(\mathrm{~ns}) \\
{[-0,2439 ; 0,12695]}\end{array}$ & $\begin{array}{c}-0,0964(\mathrm{~ns}) \\
{[-0,2956 ; 0,10285]}\end{array}$ & $\begin{array}{c}-0,0852(\mathrm{~ns}) \\
{[-0,2346 ; 0,06419]}\end{array}$ & $\begin{array}{c}0,0469(\mathrm{~ns}) \\
{[-0,1582 ; 0,25196]}\end{array}$ \\
\hline $\begin{array}{l}\text { Emploi en équivalent } \\
\text { temps plein }\end{array}$ & $\begin{array}{c}-0,2776^{\star \star *} \\
{[-0,4601 ;-0,0950]}\end{array}$ & $\begin{array}{c}-0,1301(\mathrm{~ns}) \\
{[-0,3667 ; 0,1065]}\end{array}$ & $\begin{array}{c}-0,1125(\mathrm{~ns}) \\
{[-0,2864 ; 0,0614]}\end{array}$ & $\begin{array}{c}-0,2532^{\star *} \\
{[-0,4735 ;-0,0328]}\end{array}$ \\
\hline Salaire horaire brut & $\begin{array}{c}-0,0051(\mathrm{~ns}) \\
{[-0,0998 ; 0,0895]}\end{array}$ & $\begin{array}{c}0,148^{\star \star *} \\
{[0,0435 ; 0,2526]}\end{array}$ & $\begin{array}{c}0,0479(\mathrm{~ns}) \\
{[-0,0463 ; 0,1421]}\end{array}$ & $\begin{array}{c}-0,1486^{\star \star \star} \\
{[-0,2598 ;-0,0373]}\end{array}$ \\
\hline
\end{tabular}

Champ : panel de 92939 entreprises de cinq salariés et plus au cours des années 2002 à 2005 appartenant aux secteurs d'activité privés non agricoles.

Source : bases Arome, Orme et Sequoia (Acoss), DADS et Suse (Insee).

Lecture : *** significatif au seuil de $1 \% ;{ }^{* *}$ de $5 \%$; (ns) non significatif. Les valeurs entre crochet correspondent aux bornes de l'intervalle de confiance à $95 \%$.

Lorsque l'on estime le modèle en utilisant les Moindres carrés ordinaires, l'élasticité de l'emploi en équivalent temps plein au coût du travail est de -0,2776 dans les entreprises passées aux 35 heures avant 2003. Alors que la méthode des MCO permet d'évaluer une élasticité au point moyen de l'échantillon, la régression quantile évalue cette élasticité à la médiane ou à un autre quantile de la distribution, ce qui permet de vérifier si les résultats sont robustes.

Tableau 6

Estimation de l'élasticité des variables d'intérêt à l'évolution du coût du travail pour les entreprises aux 35 heures pour différents niveaux de hausse du coût du travail

\begin{tabular}{|c|c|c|c|}
\hline Type de régression & $\mathrm{MCO}$ & Nearest Neighbor & Kernel \\
\hline \multicolumn{4}{|l|}{ Emploi brut } \\
\hline \multicolumn{4}{|l|}{ Variation du coût du travail } \\
\hline Inférieur à 0,5 & $\begin{array}{c}-0,1188(\mathrm{~ns}) \\
{[-0,3336 ; 0,0960]}\end{array}$ & $\begin{array}{c}-0,1824(\mathrm{~ns}) \\
{[-0,4889 ; 0,1240]}\end{array}$ & $\begin{array}{c}-0,1564(\mathrm{~ns}) \\
{[-0,361 ; 0,048]}\end{array}$ \\
\hline Compris entre 0,5 et 1 & $\begin{array}{c}0,0049 \text { (ns) } \\
{[-0,2483 ; 0,2582]}\end{array}$ & $\begin{array}{c}-0,3778^{\star \star} \\
{[-0,7480 ;-0,0076]}\end{array}$ & $\begin{array}{c}-0,2833(\mathrm{~ns}) \\
{[-0,592 ; 0,025]}\end{array}$ \\
\hline Compris entre 1 et 2 & $\begin{array}{c}-0,0947(\mathrm{~ns}) \\
{[-0,3921 ; 0,2027]}\end{array}$ & $\begin{array}{c}-0,3954(\mathrm{~ns}) \\
{[-0,8605 ; 0,0696]}\end{array}$ & $\begin{array}{c}-0,5536^{\star \star \star} \\
{[-0,786 ;-0,321]}\end{array}$ \\
\hline Compris entre 2 et 3 & $\begin{array}{c}-0,6163^{\star \star} \\
{[-1,1469 ;-0,0858]}\end{array}$ & $\begin{array}{c}-1,3510^{\star \star \star} \\
{[-2,0478 ;-0,6542]}\end{array}$ & $\begin{array}{c}-1,2799 * * \\
{[-1,837 ;-0,722]}\end{array}$ \\
\hline \multicolumn{4}{|c|}{ Emploi en équivalent temps plein } \\
\hline \multicolumn{4}{|l|}{ Variation du coût du travail } \\
\hline Inférieur à 0,5 & $\begin{array}{c}-0,3608^{\star \star \star} \\
{[-0,5972 ;-0,1495]}\end{array}$ & $\begin{array}{c}-0,3749^{\star \star \star} \\
{[-0,7139 ;-0,0621]}\end{array}$ & $\begin{array}{c}-0,4553^{\star \star *} \\
{[-0,743 ;-0,200]}\end{array}$ \\
\hline Compris entre 0,5 et 1 & $\begin{array}{c}-0,3855^{\star \star \star} \\
{[-0,6640 ;-0,1388]}\end{array}$ & $\begin{array}{c}-0,7243^{\star \star \star} \\
{[-1,1399 ;-0,3686]}\end{array}$ & $\begin{array}{c}-0,6298^{\star \star \star} \\
{[-0,913 ;-0,399]}\end{array}$ \\
\hline Compris entre 1 et 2 & $\begin{array}{c}-0,5905^{\star \star *} \\
{[-0,9288 ;-0,3134]}\end{array}$ & $\begin{array}{c}-0,7827^{\star \star \star} \\
{[-1,3074 ;-0,3392]}\end{array}$ & $\begin{array}{c}-0,8610^{\star \star \star} \\
{[-1,148 ;-0,664]}\end{array}$ \\
\hline Compris entre 2 et 3 & $\begin{array}{c}-1,5702^{\star \star \star} \\
{[-2,3093 ;-1,1467]}\end{array}$ & $\begin{array}{c}-2,2469^{\star \star \star} \\
{[-3,2544 ;-1,6910]}\end{array}$ & $\begin{array}{c}-2,1744^{\star \star \star} \\
{[-3,018 ;-1,768]}\end{array}$ \\
\hline \multicolumn{4}{|l|}{ Salaire horaire brut } \\
\hline \multicolumn{4}{|l|}{ Variation du coût du travail } \\
\hline Inférieur à 0,5 & $\begin{array}{c}-0,1110(\mathrm{~ns}) \\
{[-0,2231 ; 0,0010]}\end{array}$ & $\begin{array}{c}-0,0782(\mathrm{~ns}) \\
{[-0,2314 ; 0,0751]}\end{array}$ & $\begin{array}{c}-0,1303(\mathrm{~ns}) \\
{[-0,335 ; 0,074]}\end{array}$ \\
\hline Compris entre 0,5 et 1 & $\begin{array}{c}0,0058(\mathrm{~ns}) \\
{[-0,1321 ; 0,1437]}\end{array}$ & $\begin{array}{c}-0,1259 \text { (ns) } \\
{[-0,3111 ; 0,0592]}\end{array}$ & $\begin{array}{c}-0,0630(\mathrm{~ns}) \\
{[-0,186 ; 0,060]}\end{array}$ \\
\hline Compris entre 1 et 2 & $\begin{array}{c}0,6412^{\star \star \star} \\
{[0,4805 ; 0,8019]}\end{array}$ & $\begin{array}{c}0,5141^{\star \star *} \\
{[0,2816 ; 0,7466]}\end{array}$ & $\begin{array}{c}0,5932^{\star \star *} \\
{[0,438 ; 0,748]}\end{array}$ \\
\hline Compris entre 2 et 3 & $\begin{array}{c}0,7320^{\star \star *} \\
{[0,4495 ; 1,0144]}\end{array}$ & $\begin{array}{c}0,3555 \text { (ns) } \\
{[-0,0626 ; 0,7736]}\end{array}$ & $\begin{array}{c}0,4977^{\star \star \star} \\
{[0,219 ; 0,776]}\end{array}$ \\
\hline
\end{tabular}

Lecture : *** significatif au seuil de $1 \%$; ${ }^{* *}$ de $5 \%$; (ns) non significatif. Les valeurs entre crochet correspondent aux bornes de l'intervalle de confiance à $95 \%$. Lorsque l'on estime le modèle en utilisant les MCO, l'élasticité de l'emploi en équivalent temps plein au coût du travail est de - 0,3608 dans les entreprises passées aux 35 heures avant 2003 pour une variation du coût du travail inférieure à 0,5.

Champ : panel de 92939 entreprises de cinq salariés et plus au cours des années 2002 à 2005 appartenant aux secteurs d'activité privés non agricoles.

Source : bases Arome, Orme et Sequoia (Acoss), DADS et Suse (Insee). 
aux salariés à temps partiel dans ces entreprises entre 2002 et 2005. Cet effet est à imputer à la partie supérieure de la distribution de l'emploi
(- 0,25 pour le troisième quartile). Ce résultat n'est pas surprenant : consécutivement à la loi de 2003, les entreprises à 35 heures ont subi une

Tableau 7

Estimation de l'élasticité des variables d'intérêt à l'évolution du coût du travail pour les entreprises restées à 39 heures

\begin{tabular}{|c|c|c|c|c|}
\hline & \multirow[t]{2}{*}{$\mathrm{MCO}$} & \multicolumn{3}{|c|}{ Régressions quantile } \\
\hline & & $25 \%$ & $50 \%$ & $75 \%$ \\
\hline Emploi brut & $\begin{array}{c}-0,2234^{\star \star \star} \\
{[-0,3414 ;-0,1054]}\end{array}$ & $\begin{array}{c}-0,2303^{\star \star \star} \\
{[-0,3554 ;-0,1052]}\end{array}$ & $\begin{array}{c}-0,1512^{\star \star \star} \\
{[-0,2439 ;-0,0585]}\end{array}$ & $\begin{array}{c}-0,1424^{\star \star} \\
{[-0,2740 ;-0,0107]}\end{array}$ \\
\hline $\begin{array}{l}\text { Emploi en équivalent } \\
\text { temps plein }\end{array}$ & $\begin{array}{c}-0,3133^{\star \star *} \\
{[-0,4252 ;-0,2015]}\end{array}$ & $\begin{array}{c}-0,3828^{\star \star \star} \\
{[-0,5181 ;-0,2476]}\end{array}$ & $\begin{array}{c}-0,1260^{\star \star *} \\
{[-0,2259 ;-0,0261]}\end{array}$ & $\begin{array}{c}-0,1680^{\star \star *} \\
{[-0,2980 ;-0,0379]}\end{array}$ \\
\hline Salaire horaire brut & $\begin{array}{c}-0,4484^{\star \star \star} \\
{[-0,5082 ;-0,3885]}\end{array}$ & $\begin{array}{c}-0,6480^{\star \star \star} \\
{[-0,7124 ;-0,5836]}\end{array}$ & $\begin{array}{c}-0,2774^{\star \star \star} \\
{[-0,3340 ;-0,2208]}\end{array}$ & $\begin{array}{c}-0,1171^{\star \star \star} \\
{[-0,1889 ;-0,0453]}\end{array}$ \\
\hline
\end{tabular}

Lecture : se reporter au tableau $5 .{ }^{* * *}$ significatif au seuil de $1 \%$; ** de $5 \%$; (ns) non significatif. Les valeurs entre crochets correspondent aux bornes de l'intervalle de confiance à $95 \%$.

Champ : panel de 92939 entreprises de cinq salariés et plus au cours des années 2002 à 2005 appartenant aux secteurs d'activité privés non agricoles.

Source : bases Arome, Orme et Sequoia (Acoss), DADS et Suse (Insee).

Tableau 8

Estimation de l'élasticité des variables d'intérêt à l'évolution du coût du travail pour les entreprises restées à 39 heures pour différents niveaux de hausse du coût du travail

\begin{tabular}{|c|c|c|c|}
\hline Type de régression & $\mathrm{MCO}$ & Nearest Neighbor & Kernel \\
\hline \multicolumn{4}{|l|}{ Emploi brut } \\
\hline $\begin{array}{l}\text { Variation du coût du travail } \\
\text { Supérieur à }+0,05\end{array}$ & $\begin{array}{c}-0,2929^{\star \star \star} \\
{[-0,4349 ;-0,1509]}\end{array}$ & $\begin{array}{c}-0,2323^{\star \star} \\
{[-0,4275 ;-0,0372]}\end{array}$ & $\begin{array}{c}-0,2655^{\star \star \star} \\
{[-0,4282 ;-0,1029]}\end{array}$ \\
\hline Compris entre $-0,03$ et $+0,05$ & $\begin{array}{c}-0,1968^{\star \star} \\
{[-0,3828 ;-0,0109]}\end{array}$ & $\begin{array}{c}0,3671^{\star \star} \\
{[0,0313 ; 0,7029]}\end{array}$ & $\begin{array}{c}0,1958(\mathrm{~ns}) \\
{[-0,0441 ; 0,4357]}\end{array}$ \\
\hline Compris entre - 1 et $-0,03$ & $\begin{array}{c}-0,2835^{\star \star \star} \\
{[-0,4814 ;-0,0857]}\end{array}$ & $\begin{array}{c}0,2923(\mathrm{~ns}) \\
{[-0,0514 ; 0,6361]}\end{array}$ & $\begin{array}{c}0,1462(\mathrm{~ns}) \\
{[-0,1403 ; 0,4326]}\end{array}$ \\
\hline Compris entre -2 et -1 & $\begin{array}{c}-0,2418^{\star *} \\
{[-0,4819 ;-0,0018]}\end{array}$ & $\begin{array}{c}0,0769 \text { (ns) } \\
{[-0,3000: 0,4538]}\end{array}$ & $\begin{array}{c}0,1154(\mathrm{~ns}) \\
{[-0,1861: 0,4169]}\end{array}$ \\
\hline Compris entre -3 et -2 & $\begin{array}{c}-0,4630^{\star *} \\
{[-0,8677 ;-0,0583]}\end{array}$ & $\begin{array}{c}-0,3086(\mathrm{~ns}) \\
{[-0,9136 ; 0,2963]}\end{array}$ & $\begin{array}{c}-0,1852(\mathrm{~ns}) \\
{[-0,5481 ; 0,1778]}\end{array}$ \\
\hline \multicolumn{4}{|l|}{ Emploi en équivalent temps plein } \\
\hline \\
\hline Supérieur à + 0,05 & $\begin{array}{c}-0,3275^{\star \star \star} \\
{[-0,4637 ;-0,1913]}\end{array}$ & $\begin{array}{c}-0,2489^{\star \star \star} \\
{[-0,4441 ;-0,0538]}\end{array}$ & $\begin{array}{c}-0,2489^{\star \star \star} \\
{[-0,3790 ;-0,1188]}\end{array}$ \\
\hline Compris entre $-0,03$ et $+0,05$ & $\begin{array}{c}-0,0819(\mathrm{~ns}) \\
{[-0,2635 ; 0,0996]}\end{array}$ & $\begin{array}{c}-0,0245(\mathrm{~ns}) \\
{[-0,3603 ; 0,3113]}\end{array}$ & $\begin{array}{c}0,0734(\mathrm{~ns}) \\
{[-0,2144 ; 0,3613]}\end{array}$ \\
\hline Compris entre - 1 et $-0,03$ & $\begin{array}{c}-0,3057^{\star \star *} \\
{[-0,4974 ;-0,1140]}\end{array}$ & $\begin{array}{c}-0,2046(\mathrm{~ns}) \\
{[-0,5484 ; 0,1391]}\end{array}$ & $\begin{array}{c}-0,1754(\mathrm{~ns}) \\
{[-0,4046 ; 0,0538]}\end{array}$ \\
\hline Compris entre - 2 et - 1 & $\begin{array}{c}-0,2740^{\star \star} \\
{[-0,5061 ;-0,0420]}\end{array}$ & $\begin{array}{c}-0,3846^{\star \star} \\
{[-0,7615 ;-0,0077]}\end{array}$ & $\begin{array}{c}-0,1923(\mathrm{~ns}) \\
{[-0,4938 ; 0,1092]}\end{array}$ \\
\hline Compris entre -3 et -2 & $\begin{array}{c}-0,3974^{\star *} \\
{[-0,7861 ;-0,0087]}\end{array}$ & $\begin{array}{c}-0,5556^{\star \star} \\
{[-1,1605 ;-0,005]}\end{array}$ & $\begin{array}{c}-0,3086(\mathrm{~ns}) \\
{[-0,7926 ; 0,1753]}\end{array}$ \\
\hline \multicolumn{4}{|l|}{ Salaire horaire brut } \\
\hline \multirow{2}{*}{$\begin{array}{l}\text { Variation du coût du travail } \\
\text { Supérieur à }+0,05\end{array}$} & & & \\
\hline & $\begin{array}{c}-0,3371^{\star \star \star} \\
{[-0,4107 ;-0,2635]}\end{array}$ & $\begin{array}{c}-0,2323^{\star \star \star} \\
{[-0,3299 ;-0,1348]}\end{array}$ & $\begin{array}{c}-0,2489^{\star \star \star} \\
{[-0,3368 ;-0,1611]}\end{array}$ \\
\hline Compris entre $-0,03$ et $+0,05$ & $\begin{array}{c}-0,8979^{\star \star \star} \\
{[-1,0025 ;-0,7932]}\end{array}$ & $\begin{array}{c}-0,9545^{\star \star \star} \\
{[-1,1464 ;-0,7627]}\end{array}$ & $\begin{array}{c}-1,0280^{\star \star \star} \\
{[-1,2342 ;-0,8217]}\end{array}$ \\
\hline Compris entre - 1 et $-0,03$ & $\begin{array}{c}-0,9315^{\star \star \star} \\
{[-1,0375 ;-0,8255]}\end{array}$ & $\begin{array}{c}-1,1108^{\star \star *} \\
{[-1,2827 ;-0,9390]}\end{array}$ & $\begin{array}{c}-1,0816^{\star \star \star} \\
{[-1,2821 ;-0,8811]}\end{array}$ \\
\hline Compris entre -2 et -1 & $\begin{array}{c}-0,8388^{\star \star \star} \\
{[-0,9619 ;-0,7157]}\end{array}$ & $\begin{array}{c}-0,9230^{\star \star \star} \\
{[-1,0737 ;-0,7722]}\end{array}$ & $\begin{array}{c}-0,9999^{\star \star *} \\
{[-1,1808 ;-0,8190]}\end{array}$ \\
\hline Compris entre - 3 et -2 & $\begin{array}{c}-0,7947^{\star \star \star} \\
{[-0,9952 ;-0,5942]}\end{array}$ & $\begin{array}{c}-0,9259^{\star \star \star} \\
{[-1,1679 ;-0,6840]}\end{array}$ & $\begin{array}{c}-0,9259^{\star \star *} \\
{[-1,2284 ;-0,6235]}\end{array}$ \\
\hline
\end{tabular}

Lecture : se reporter au tableau $6 .{ }^{* *}$ significatif au seuil de $1 \% ;{ }^{* *}$ de $5 \%$; (ns) non significatif. Les valeurs entre crochet correspondent aux bornes de l'intervalle de confiance à $95 \%$.

Champ : panel de 92939 entreprises de cinq salariés et plus au cours des années 2002 à 2005 appartenant aux secteurs d'activité privés non agricoles.

Source : bases Arome, Orme et Sequoia (Acoss), DADS et Suse (Insee). 
baisse des exonérations sur les salaires supérieurs à 1,7 Smic.

On détaille le processus de calcul des élasticités spécifiques à chaque groupe d'entreprises traitées en prenant pour exemple les entreprises passées aux 35 heures avec une hausse du coût du travail comprise entre $0,5 \%$ et $1 \%$. Ces entreprises connaissent une baisse relative du coût du travail de 3,17 point de pourcentage par rapport au groupe témoin (c'està-dire le groupe des entreprises passées aux 35 heures avec une variation du coût du travail supérieure à $+3 \%$ - se reporter à l'encadré 2 ). Pour ce groupe de traitement, l'impact de la réforme Fillon sur l'emploi en équivalent temps plein a été respectivement de 1,22, 2,3 et 2,0 point selon la méthode économétrique utilisée (respectivement MCO, Nearest Neighbor, Kernel) (cf. annexe 4). En divisant chacun de ces trois coefficients par - 0,0317 , on obtient l'élasticité spécifique (respectivement - 0,38, - 0,72 et - 0,62 selon la méthode économétrique) (cf. tableau 6). Par construction, il n'est pas possible de calculer une élasticité spécifique pour le groupe témoin.

L'effet de la réforme de 2003 est négatif sur l'emploi des entreprises passées à 35 heures, mais uniquement pour les entreprises qui ont expérimenté une forte augmentation du coût du travail (entre $2 \%$ et $3 \%$ ) (cf. tableau 6). Cet impact est, rappelons-le, principalement le fait des entreprises embauchant sur des postes à salaire assez élevé : comme indiqué plus haut, la part des salariés percevant plus de 1,7 Smic était de 44,65\% dans les entreprises ayant subi une hausse du coût du travail comprise entre $2 \%$ et $3 \%$ (cf. tableau 3).

La sensibilité de l'emploi au coût du travail semble être croissante en valeur absolue avec l'ampleur de la variation subie : elle est plus faible dans les entreprises où le coût du travail a le moins augmenté (sensibilité de $-0,45$ à $-0,36$, pour une variation inférieure à $+0,5 \%$; au contraire, sensibilité de $-2,25$ à $-1,57$, pour une variation de $+2 \%$ à $+3 \%$ ).

Les effets sont moins significatifs sur les salaires. Les élasticités significatives sont plutôt de signe positif. La hausse du coût du travail aurait donc été de pair avec une hausse des salaires bruts. Ce mouvement des salaires dans les entreprises passées à 35 heures est lié principalement aux entreprises dans lesquelles la hausse du coût du travail générée par le dispositif Fillon a été la plus élevée $(+1 \%$ à $+3 \%)$.

\section{Entreprises à 39 heures : la réforme de 2003 a conduit à une hausse de l'emploi et des salaires}

Concernant les entreprises qui ne sont pas passées aux 35 heures, deux résultats principaux s'imposent. Tout d'abord, l'élasticité de l'emploi (brut ou en équivalent temps plein) au coût du travail est bien négative $(-0,22$ et $-0,31$, cf. tableau 7). Les entreprises restées à 39 heures ayant connu une baisse du coût moyen du travail lors de la mise en place des mesures Fillon, l'emploi a augmenté, tant en heures qu'en effectifs salariés. La plus forte croissance de l'emploi (en effectifs ou en heures travaillées) intervient dans les entreprises qui ont connu les plus fortes hausses d'exonérations (variation du coût du travail comprise entre $-3 \%$ et $-2 \%$ ).

Ensuite, la mise en place de la loi Fillon a plutôt eu un effet d'entraînement sur les salaires, comme dans les entreprises passées à 35 heures. L'élasticité des salaires au coût du travail est cette fois-ci négative (de l'ordre de - 0,44) et le coût du travail a globalement baissé ce qui conduit bien au total à une hausse des salaires. Ces entreprises emploient une large proportion de salariés rémunérés entre 1,2 et $1,7 \mathrm{Smic}$, pour lesquels les effets de diffusion des hausses successives du Smic horaire ont pu se faire sentir entre 2002 et 2005 (6).

\section{La réforme de 2003 n'aurait eu qu'un impact indécis, ou même légèrement négatif, sur l'emploi total}

L'effet global de la réforme sur l'emploi a été très faible, et même peut-être légèrement négatif. Dans les entreprises restées à 39 heures, le coût du travail a diminué grâce aux allègements de cotisations sociales et malgré la hausse du salaire minimum : elles ont dans l'ensemble bénéficié de cette réforme. En revanche, les entreprises à 35 heures ont subi une hausse du coût du travail liée principalement à la perte des allègements octroyés pour les salariés rémunérés au-delà de 1,7 Smic, à la fin des aides incitatives prévues dans le cadre de la loi Aubry 1 et à la hausse des garanties mensuelles de rémunération (pour les entreprises de 20 salariés et plus).

6. Une estimation complémentaire proposée par Bunel et al (2009), montre que la part des non qualifiés dans l'effectif total des entreprises a eu tendance à augmenter dans les entreprises demeurées à 39 heures (notamment pour celles dont le coût du travail a le plus baissé) et à diminuer pour les entreprises à 35 heures. 
Cette hausse du coût du travail a conduit à une baisse de l'emploi de faible intensité.

On peut tenter de préciser l'ordre de grandeur de ces effets. Concernant les entreprises demeurées à 39 heures, l'effet global sur l'emploi brut dépend de l'évolution moyenne du coût du travail (- 1,017\%) et de l'élasticité par rapport au coût du travail $(-0,2234)$. On obtient un effet de l'ordre de 0,24\% représentant 3000 emplois environ (7). Même si l'on extrapolait ce résultat à l'ensemble de la population active, c'est-à-dire en considérant les 15 millions de salariés du secteur privé marchand dont $46 \%$ sont embauchés par des entreprises à 39 heures, on obtiendrait un effet sur l'emploi de l'ordre de 15500 emplois créés ou sauvegardés par la réforme Fillon. Un exercice similaire peut être effectué pour l'emploi en équivalent temps plein. Dans ce cas, l'impact sur l'emploi est légèrement plus important de l'ordre de 4300 emplois dans l'échantillon et de 21500 emplois par extrapolation.

Concernant les entreprises à 35 heures, l'évolution du coût du travail a été de $+1,71 \%$ en moyenne (moyenne pondérée par les effectifs).

7. Cet effet moyen est obtenu en calculant une moyenne pondérée fonction de la part des entreprises dans l'emploi.
Comme l'élasticité de l'emploi en équivalent temps plein par rapport à ce coût est de - 0,278, l'effet global est négatif. Il est de l'ordre de - 0,48 \% soit 7700 emplois en moins environ. En extrapolant à l'ensemble de la population (54\% des 15 millions de salariés du secteur marchand sont embauchés par des entreprises restées à 35 heures), on obtiendrait un effet de 39000 emplois détruits.

Ainsi, l'effet global sur l'emploi de la réforme de 2003, concernant à la fois sur les entreprises à 35 heures et celles à 39 heures, semble avoir été très faible, voire légèrement négatif. Il s'agit là d'un résultat valant en moyenne pour les deux groupes d'entreprises et il n'est pas incompatible avec le fait que la réforme ait pu exercer un impact significatif sur certaines entreprises dans quelques secteurs d'activité particuliers.

Rappelons que cet article s'inscrit dans une démarche d'évaluation micro-économétrique dans le prolongement de Crépon et Desplatz (2001) et ne s'appuie sur aucun bouclage macro-économique. Notre évaluation ne prend en compte ni les effets volume, ni les effets de substitution intersectoriels. Enfin, elle ne précise pas l'impact de cette réforme par niveaux de qualification et de salaire.

\section{BIBLIOGRAPHIE}

Acoss (2005), « Situations contrastées pour les entreprises lors du passage aux allègements de la loi Fillon », Acoss-Stat, $\mathrm{n}^{\circ} 22$, janvier.

Beach C. et Balfour F. (1983), « Estimated Payroll Tax Incidence and Aggregate Demand for Labour in the United-Kingdom », Economica, n ${ }^{\circ} 50$, pp. 35-48.

Bell B. et Nickell S. (1997), « Would Cutting Payroll Taxes on the Unskilled have a Significant Impact on Unemployment? ", in D.J. Snower and G. de la Dehesa (eds.), Unemployment Policy : Government Options for the Labour Market, Cambridge University Press.

Belleville A. et Saint-Martin A. (2002), «Emplois aidés et performances des entreprises », Premières synthèses, $\mathrm{n}^{\circ} 02.1$.

Berry J.B. (2008), « Les bénéficiaires de la revalorisation du Smic au $1^{\mathrm{er}}$ juillet 2007 », Premières synthèses, $\mathrm{n}^{\circ} 10.3$.
Brittain J. (1971), « The Incidence of Social Security Payroll Taxes », American Economic Review, n ${ }^{\circ} 61$, vol. 1, pp. 110-125.

Brodaty T., Crépon B. et Fougère D. (2000), « Using Matching Estimators to evaluate Alternative Youth Employment Programs : Evidence from France, 1986-1988 », Document de travail du C.E.P.R, $\mathrm{n}{ }^{\circ} 2604$.

Bunel M. (2005), « Aides incitatives et déterminants des embauches des établissements passés à 35 heures », Économie et Statistique, n 376-377, pp. 91-115.

Bunel M., Gilles F. et L'Horty Y. (2009), « Les effets des allègements de cotisations sociales sur l'emploi et les salaires : une évaluation de la réforme Fillon de 2003 », Document travail du $C E E, \mathrm{n}^{\circ} 122$.

Bur Y. (2008), « Mission d'information commune sur les exonérations de cotisations sociales ", Rapport d'information de l'Assemblée Nationale. 
Burnod G. et Chenu A. (2001), « Employés qualifiés et non qualifiés : une proposition d'aménagement de la nomenclature des catégories socioprofessionnelles », Travail et Emploi, $\mathrm{n}^{\circ} 86$, pp. 87-105.

Chéron A., Hairault J.-O. et Langot F. (2008), «A Quantitative Evaluation of Payroll Tax Subsidies for Low-Wage Workers : An Equilibrium Search Framework », Journal of Public Economics, ${ }^{\circ}$ 92, vol. 3-4, pp. 817-843.

Crépon B. et Desplatz R. (2001), « Une nouvelle évaluation des effets des allègements de charges sociales sur les bas salaires ", Économie et Statistique, $\mathrm{n}^{\circ} 348$, pp. 1-22.

CSERC (1996), L'allègement des charges sociales sur les bas salaires, Rapport au Premier Ministre, La Documentation Française.

Insee (2007), DADS, Guide méthodologique, validité 2005 .

Fitoussi J.-P. (2000), « Payroll Tax Reductions for the Low Paid », OECD Economic Studies, $\mathrm{n}^{\circ} 31$, pp. 115-131.

Gafsi I., L'Horty Y. et Mihoubi F. (2005), « Vingt ans d'évolution de l'emploi peu qualifié et du coût du travail : des ruptures qui coïncident ? », Revue française d'Économie, n 19, vol. 3, pp. 91-116.

Gilles F. (2006), « Quels effets des réorganisations sur la date de passage aux 35 heures ? Une étude sur données individuelles d'entreprises », Revue Économique, $\mathrm{n}^{\circ}$ 57, vol. 6, pp. 1401-1426.

Gubian A., Jugnot S., Lerais F. et Passeron V. (2004), « Les effets de la RTT sur l'emploi : des simulations ex ante aux évaluations ex post ", Économie et statistique, ${ }^{\circ}$ 376-377, pp. 25-54.

Gruber J. (1997), « The Incidence of Payroll Taxation : Evidence from Chile », Journal of Labor Economics, $\mathrm{n}^{\circ}$ 15, vol. 3, S72-S101.

Hamermesh D. (1979), « New Estimates of the Incidence of the Payroll Tax », Southern Economic Journal, $\mathrm{n}^{\circ} 45$, pp. 1208-19.

Heckman J., Ichimura H. et Todd P. (1998), « Matching as an Econometric Estimator », Review of Economic Studies, n ${ }^{\circ}$ 65, pp. 261-294.

Jamet S. (2005), «Allègements généraux de cotisations sociales et emploi peu qualifié : de l'impact sectoriel à l'effet macro-économique », Revue Française d'Économie, n 19 , pp. 57-90.
Koenker R. et Bassett G. (1978), « Regression Quantiles », Econometrica, vol. 46, n 1, p. 33-50.

Koenker R. et Hallock K. (2001), « Regression Quantiles », Journal of Economic Perspectives, vol. $15, n^{\circ} 4$, p. 143-156.

Koubi M. et Lhommeau B. (2007), « Les effets de diffusion de court terme des hausses du Smic dans les grilles salariales des entreprises de dix salariés ou plus sur la période 2000-2005 », les salaires en France, Insee.

Kramarz F. et Philippon T. (2001), " The Impact of Differential Payroll Tax Subsidies on Minimum Wage Employment », Journal of Public Economics, ${ }^{\circ} 82$, p. 115-146.

Kugler A. et Kugler M. (2008), « Labor Market Effects of Payroll Taxes in Developing Countries : Evidence from Colombia », NBER working paper 13855, March.

Lechner M. (1999), «Identification and Estimation of Causal Effects of Multiple Treatments Under the Conditional Independence Assumption », IZA discussion paper 91.

L'Horty Y. (2000), « Quand les hausses du Smic réduisent le coût du travail », Revue économique, vol. $51, \mathrm{n}^{\circ} 3$, mai 2000 .

Marx I. (2005), « Job Subsidies and Cuts in Employers' Social Security Contributions : the Verdict of Empirical Evaluation Studies », Working paper, University of Michigan.

Montaut A. (2008), « Une heure de travail salarié en 2004 deux fois plus chère mais deux fois plus productive qu'en 1975 », Insee Première, $\mathrm{n}^{\circ} 1214$.

OECD (2003), Employment Outlook, Paris : OECD.

Seguin S. (2006), « Les bénéficiaires de la revalorisation du Smic et des garanties mensuelles au

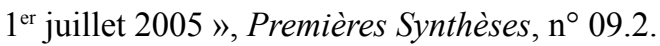

Sianesi B. et Leuven E. (2003), «PSmatch2 : Stata Module to Perform Full Mahalanobis and Propensity Score Matching, Common Support Graphing and Covariate Imbalance Testing », EconPapers. 
Stewart M.B. (1983), « On Least Squares Estimation When the Dependent Variable is Grouped ", Review of Economic Studies, vol. 50, $\mathrm{n}^{\circ} 4$, p. 737-753.
Vroman W. (1974a), « Employer Payroll Tax Incidence : Empirical Tests with Cross-Country Data », Finances Publiques, n 24, p. 184-200. 
En 1998 et en 2000 ont été adoptées deux lois visant à organiser le passage aux 35 heures en réduisant la durée légale hebdomadaire de travail de 39 heures à 35 heures. II s'agit des lois dites Aubry 1 (13 juin 1998) et Aubry 2 (19 janvier 2000). Le but affiché de ces deux lois était d'inciter les entreprises à réduire le temps de travail de leurs salariés pour favoriser la création d'emplois et réduire le chômage.

La loi «Aubry 1 » (loi n 1998 - 461) prévoyait de réduire la durée légale de la semaine de travail de 39 à 35 heures, à compter du $1^{\text {er }}$ janvier 2000 pour les entreprises de plus de 20 salariés et du $1^{\mathrm{er}}$ janvier 2002 pour les autres. Les entreprises qui mettaient en place une réduction du temps de travail avant l'entrée en vigueur de la loi pouvaient bénéficier d'aides dites « incitatives " dès qu'elles s'engageaient à la fois à baisser de $10 \%$ la durée effective du travail hebdomadaire et à créer des emplois à hauteur de $6 \%$ des effectifs initiaux (accords offensifs) ou à ne pas détruire des emplois à hauteur de $6 \%$ des effectifs initiaux (accords défensifs) dans le cadre de plans de licenciement engagés avant le passage aux 35 heures. Ces aides incitatives étaient perçues sous la forme de réductions des cotisations patronales, correspondant à une somme fixe pour chaque salarié employé et concerné par la RTT. Ces aides étaient versées sur une période de cinq ans. Leur montant dépendait de la date de signature de l'accord par l'entreprise ou l'établissement. Si l'accord était signé entre ler juin 1998 et juin 1999, l'entreprise bénéficie de 1372 euros (9 000 francs) par salarié la première année, ce montant diminuant de 150 euros (1 000 francs) chaque année ; si l'accord était signé entre juin et décembre 1999, l'entreprise touchait 1067 euros (7 000 francs) la première année, 915 euros (6 000 francs) l'année suivante et ainsi de suite. Des réductions de cotisations supplémentaires pouvaient être accordées si l'entreprise réduisait la durée de travail effective de $15 \%$ ou plus et si elle s'engageait à augmenter son niveau d'emploi initial de $9 \%$.

La loi Aubry 2 (loi n²000-37) a confirmé la réduction de la durée légale à 35 heures hebdomadaires ou 1600 heu- res annuelles dès le $1^{\text {er }}$ janvier 2000 pour les entreprises employant plus de 20 salariés. Les entreprises employant plus de 20 salariés signant un accord après le $1^{\text {er }}$ janvier 2000 bénéficiaient d'une aide pérenne, baisse unique de cotisations patronales non conditionnelle à des engagements en termes de créations d'emplois ou d'effectivité de la RTT (aucune obligation en terme de mode de décompte du temps de travail). Ce nouveau dispositif d'allégement de cotisations (sur les bas et moyens salaires) - ou aide structurelle Aubry 2 - était décroissant avec le salaire jusqu'à 1,7 Smic.

Pour les entreprises employant plus de 20 salariés mais ayant signé un accord avant le $1^{\mathrm{er}}$ janvier 2000 et ayant bénéficié des aides incitatives avant cette date, la baisse de cotisations est la somme des aides Aubry 2 et des aides incitatives Aubry 1 cumulables. La durée de ce cumul est au maximum de cinq ans. Toutefois à partir du second semestre 2004, c'est-à-dire après l'adoption de la réforme de 2003, les entreprises devaient choisir entre le dispositif Aubry 1 et le dispositif «Fillon-suiteà-Aubry 2 »

Pour les entreprises de 20 salariés et moins, ces aides incitatives pouvaient être perçues si elles s'engageaient sur une RTT et des créations (ou non destructions) d'emplois après le $1^{\mathrm{er}}$ janvier 2002 dans les mêmes conditions que celles précédemment prévues pour les entreprises de plus de 20 salariés passées à 35 heures avant cette date.

La loi du 17 janvier 2003 relative « aux salaires, au temps de travail et au développement de l'emploi $»\left(\mathrm{n}^{\circ} 2003-\right.$ 47), a unifié tous ces dispositifs d'exonération de cotisations sociales avec ceux qui existaient antérieurement pour les entreprises à 39 heures. Elle a également unifié le Smic horaire et les Garanties mensuelles de rémunération (GMR) dont le montant était variable selon la date d'entrée dans les dispositifs Aubry (cf. tableau A). La loi a organisé la fusion des rémunérations minimales et celle des dispositifs d'exonération de façon progressive, en aménageant plusieurs étapes qui sont détaillées dans le tableau B.

\section{Tableau A}

Niveau et évolution de la GMR et du Smic horaire lors de la réforme de 2003

\begin{tabular}{|l|c|c|c|c|c|}
\hline & Juillet-01 & Juillet-02 & Juillet-03 & Juillet-04 & Juillet-05 \\
\hline IPC (en \%) & & 1,63 & 1,89 & 2,32 & 1,72 \\
\hline Smic horaire brut & 6,67 & 6,83 & 7,19 & 7,61 & 5,84 \\
& & 2,40 & 5,27 & 1,03 \\
\hline GMR1 & 1081,21 & 1100,67 & 1136,15 & 1178,54 & 1217,88 \\
(RTT entre 15/06/98 et 30/06/99) & & 1,80 & 3,22 & 3,73 & 3,34 \\
\hline GMR2 & 1094,65 & 1114,35 & 1145,54 & 1183,40 & 1217,88 \\
(RTT entre 1/07/99 et 30/06/00) & & 1,80 & 2,80 & 3,30 & 2,91 \\
\hline GMR3 & 1113,45 & 1133,49 & 1158,62 & 1190,14 & 1217,88 \\
(RTT entre 1/07/00 et 30/06/01) & & 1,80 & 2,22 & 2,72 & 2,33 \\
\hline GMR4 & 1127,23 & 1147,52 & 1168,16 & 1195,03 & 1217,88 \\
(RTT entre 1/07/01 et 30/06/02) & & 1,80 & 1,80 & 2,30 & 1,91 \\
\hline GMR5 & & 1154,27 & 1172,74 & 1197,37 & 1217,88 \\
(RTT après 1/07/02) & & 1,60 & 2,10 \\
\hline
\end{tabular}

Lecture : les grandeurs sont exprimées en euros courants. IPC : Indice des prix à la consommation. Les nombres en italiques figurant sous les montants sont les pourcentages d'évolution. Le Smic horaire a été relevé de 6,67 à 6,83 euros entre juillet 2001 et juillet 2002 , ce qui représente une hausse de $2,4 \%$ du salaire minimum.

Source : Légifrance et Insee. 
Tableau B

Évolution des barèmes d'allègements de cotisations sociales associée à la réforme de 2003 sur la période (2003 - 2005)

\begin{tabular}{|c|c|c|}
\hline Périodes & $\begin{array}{l}\text { Employeur ouvrant droit au } 30 \text { juin } 2003 \\
\text { à l'allègement } 35 \text { heures }\end{array}$ & Autre employeur \\
\hline Avant le 30 juin 2003 & $\begin{array}{l}\text { 1. Réduction maximale : } 26 \% \text { du salaire } \\
\text { brut dégressive jusqu'à } 1,7 \text { fois Smic puis } \\
\text { stable ensuite }\end{array}$ & $\begin{array}{l}\text { 2. Réduction maximale : } 18,6 \% \text { du salaire } \\
\text { brut. Limite : } 1,3 \text { fois }\end{array}$ \\
\hline 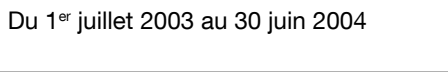 & \multirow{2}{*}{$\begin{array}{l}\text { 3. Réduction maximale : } 26 \% \text { du salaire } \\
\text { brut. Limite : } 1,7 \text { fois la garantie de rému- } \\
\text { nération applicable au } 1^{\text {er janvier } 2000} \\
\text { (GMR2) }\end{array}$} & $\begin{array}{l}\text { 4. Réduction maximale : } 20,8 \% \text { du salaire } \\
\text { brut. Limite : } 1,5 \mathrm{Smic}\end{array}$ \\
\hline Du 1 1er juillet 2004 au 31 décembre 2004 & & \multirow{2}{*}{$\begin{array}{l}\text { 5. Réduction maximale : } 23,4 \% \text { du salaire } \\
\text { brut. Limite : } 1,6 \mathrm{Smic}\end{array}$} \\
\hline Du $1^{\text {er }}$ janvier 2005 au 30 juin 2005 & $\begin{array}{l}\text { 6. Réduction maximale : } 26 \% \text { du salaire } \\
\text { brut. Limite : } 1,6 \text { fois la GMR2 }\end{array}$ & \\
\hline 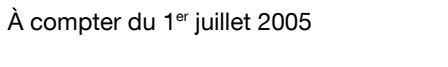 & \multicolumn{2}{|c|}{$\begin{array}{l}\text { 7. Réduction maximale : } 26 \% \text { du salaire brut } \\
\text { Limite : } 1,6 \mathrm{Smic}\end{array}$} \\
\hline
\end{tabular}




\section{CARACTÉRISTIQUES DES ENTREPRISES DE L'ÉCHANTILLON EN 2002}

En \%

\begin{tabular}{|c|c|c|c|c|}
\hline & \multicolumn{2}{|c|}{ Entreprises aux 39 heures } & \multicolumn{2}{|c|}{ Entreprises aux 35 heures } \\
\hline & Nombre & Effectif salarié & Nombre & Effectif salarié \\
\hline $\begin{array}{l}\text { Caractéristiques de la main d'œuvre } \\
\text { Effectif moyen (en nombre) } \\
\text { De } 5 \text { à } 9 \text { salariés } \\
\text { De } 10 \text { à } 19 \text { salariés } \\
\text { De } 20 \text { à } 49 \text { salariés } \\
\text { De } 50 \text { à } 199 \text { salariés } \\
\text { Plus de } 200 \text { salariés } \\
\text { Part des femmes dans l'effectif salarié } \\
\text { Part des salariés à temps partiel } \\
\text { Au moins } 30 \% \text { de la main-d'œuvre est constituée } \\
\text { de salariés non qualifiés } \\
\text { Au moins } 20 \% \text { de la main-d'œuvre est constituée } \\
\text { de salariés très qualifiés }\end{array}$ & $\begin{array}{r}40,4 \\
33,2 \\
20,5 \\
4,8 \\
1,1 \\
28,0 \\
16,9 \\
28,0 \\
50,9\end{array}$ & $\begin{array}{l}22,65 \\
10,70 \\
16,80 \\
24,10 \\
16,90 \\
31,60 \\
26,90 \\
11,80 \\
23,80 \\
60,90\end{array}$ & $\begin{array}{r}32,0 \\
24,5 \\
26,7 \\
12,1 \\
4,7 \\
38,4 \\
20,4 \\
\\
33,25 \\
58,58\end{array}$ & $\begin{array}{r}50,21 \\
3,50 \\
5,30 \\
14,70 \\
20,10 \\
56,40 \\
31,10 \\
13,80 \\
30,70 \\
67,80\end{array}$ \\
\hline $\begin{array}{l}\text { Secteur d'activité } \\
\text { Industrie } \\
\text { Construction } \\
\text { Commerce } \\
\text { Transports } \\
\text { Activités financières et immobilières et services } \\
\text { aux entreprises } \\
\text { Autres services }\end{array}$ & $\begin{array}{r}27,50 \\
22,3 \\
28,5 \\
8,2 \\
\\
11,2 \\
2,5\end{array}$ & $\begin{array}{r}39,90 \\
16,10 \\
22,00 \\
9,50 \\
\\
11,20 \\
1,30\end{array}$ & $\begin{array}{c}33,03 \\
13,6 \\
33,6 \\
3,1 \\
12,70 \\
3,9\end{array}$ & $\begin{array}{r}49,90 \\
8,50 \\
21,70 \\
6,60 \\
\\
12,20 \\
1,10\end{array}$ \\
\hline $\begin{array}{l}\text { Taux d'entrée } \\
\text { Taux de sortie } \\
\text { Indice d'Herfindhal (1) } \\
\text { Taux d'exportation }\end{array}$ & $\begin{array}{r}13,9 \\
13,3 \\
1,8 \\
8,2\end{array}$ & $\begin{array}{r}13,00 \\
12,70 \\
4,70 \\
14,30\end{array}$ & $\begin{array}{r}13,0 \\
12,3 \\
2,5 \\
9,3\end{array}$ & $\begin{array}{r}12,50 \\
12,40 \\
6,40 \\
16,30\end{array}$ \\
\hline $\begin{array}{l}\text { Autres caractéristiques } \\
\text { Appartenance à un groupe } \\
\text { Localisation en lle de France } \\
\text { Log(Intensité capitalistique) }\end{array}$ & $\begin{array}{r}17,3 \\
18,0 \\
321,3\end{array}$ & $\begin{array}{r}53,50 \\
23,00 \\
3,71\end{array}$ & $\begin{array}{r}28,84 \\
11,10 \\
338,55\end{array}$ & $\begin{array}{r}77,00 \\
15,90 \\
3,82\end{array}$ \\
\hline $\begin{array}{l}\text { Variables comptables et financières } \\
\text { Niveau d'endettement en } 2002 \\
\text { Log(valeur ajoutée en 2002) } \\
\text { Rentabilité économique en } 2002 \\
\text { Taux de marge en } 2002 \\
\text { Log(coût du travail par tête en 2002) }\end{array}$ & $\begin{array}{c}13,1 \\
637,5 \\
32,5 \\
19,7 \\
9,66\end{array}$ & $\begin{array}{c}12,40 \\
8,457 \\
25,30 \\
20,20 \\
9,71\end{array}$ & $\begin{array}{r}14,72 \\
670,78 \\
29,09 \\
21,47 \\
9,61\end{array}$ & $\begin{array}{c}11,60 \\
9,469 \\
20,50 \\
21,50 \\
9,62\end{array}$ \\
\hline $\begin{array}{l}\text { Date de passage aux } 35 \text { heures } \\
\text { Entre le } 15 / 06 / 98 \text { et le } 30 / 06 / 99 \\
\text { Entre le } 1 / 07 / 99 \text { et le } 30 / 06 / 00 \\
\text { Entre le } 1 / 07 / 00 \text { et le } 30 / 06 / 01 \\
\text { Entre le } 1 / 07 / 01 \text { et le } 30 / 06 / 02 \\
\text { Après le } 1 / 07 / 02 \\
\text { Indéterminé }\end{array}$ & & & $\begin{array}{r}2,19 \\
18,49 \\
23,97 \\
45,12 \\
6,11 \\
4,11\end{array}$ & $\begin{array}{r}2,98 \\
26,66 \\
21,83 \\
16,44 \\
2,70 \\
29,40\end{array}$ \\
\hline Nombre d'observations & 60517 & & & 32422 \\
\hline
\end{tabular}

Lecture : en 2002, (a) 40,4\% des entreprises à 39 heures comptent entre 5 et 10 salariés, ce qui représente 10,7 \% des salariés employés par ces entreprises ; (b) 33,03\% des entreprises à 35 heures sont issues des secteurs de l'industrie et emploient 49,9\% des salariés travaillant dans les entreprises aux 35 heures.

Champ : panel de 92939 entreprises de cinq salariés et plus sur les années 2002 à 2005 issues des secteurs d'activité privés non agricoles.

Source : bases Arome, Orme et Sequoia (Acoss), DADS et Suse (Insee). 


\section{MESURER L'IMPACT DE LA RÉFORME DE 2003 SUR LE COÛT DU TRAVAIL}

Pour déterminer l'évolution du coût du travail liée exclusivement à la réforme de 2003, il est nécessaire de déterminer l'évolution des aides perçues indépendamment du comportement des entreprises en termes de salaire et de structure de leur main-d'œuvre.

Pour ce faire, on calcule le taux d'exonération des entreprises en procédant en quatre étapes.

1 - On récupère à partir des DADS la distribution de la main-d'œuvre en fonction de huit tranches de salaires fonction du salaire horaire brut $([0,8-1,02[;[1,02-1,1[$; [1,1-1,2[ ; [1,2-1,3[ ; [1,3-1,4[ ; [1,4-1,5[ ; [1,5-1,6[ ; [1,61,7 [ et [ 1,7 et plus). On approche ensuite le salaire moyen versé à chacune de ces catégories au moyen du centre de classe. On calcule un poids relatif de chacune de ces tranches par rapport à la masse salariale totale.

2 - On répartit le salaire brut trimestriel issu de la base Acoss entre ces huit catégories de salariés en fonction du poids relatif calculé à l'étape 1 . Un salaire brut trimestriel est ainsi obtenu pour chacune des tranches.

3 - On applique les barèmes d'allègement de juillet 2002 pour calculer, pour chaque tranche, des montants d'exonérations et de cotisations sociales. En sommant ces informations, on calcule un taux d'exonération apparent (TEA) initial pour l'année 2002.

4 - Pour calculer le TEA virtuel pour l'année 2005, on se réfère aux barèmes d'allègement de janvier 2005, et aux salaires bruts du second trimestre 2002 des huit catégories de main-d'œuvre déterminées à l'étape 2 . On obtient ainsi les montants virtuels d'exonérations et de cotisations sociales si la structure des salaires et de l'emploi n'avait pas évolué entre 2002 et 2005. En sommant ces informations, on calcule un TEA virtuel pour l'année 2005.

Au cours des étapes 1 à 3 , dans la majorité des cas, le montant total des exonérations obtenues pour le second trimestre 2002 est inférieur à celui observé dans la base
Acoss. Cet écart est notamment de plus de $50 \%$ pour les entreprises restées aux 35 heures. Cette situation s'explique par l'écart existant entre les éléments pris en compte dans la définition du Smic et ceux retenus dans la base DADS. Les rémunérations qui ont permis de classer les salariés par tranche de Smic intègrent notamment les majorations pour heures supplémentaires, les majorations pour travail du dimanche, des jours fériés et de nuit, les primes d'ancienneté, l'intéressement et la participation. Cet écart conduit à classer trop de salariés dans les tranches hautes de la distribution des salaires.

Comme le souligne Seguin (2006), selon l'enquête sur la structure des salaires de 2002, $26 \%$ des salariés au Smic perçoivent une rémunération horaire supérieure à 1,3 Smic. Ce pourcentage est même de $30 \%$ pour les salariés à temps complet. Ces salariés sont considérés à tort comme non éligibles aux aides par les données tirées des DADS.

Par ailleurs, la structure des rémunérations peut également affecter les résultats. Comme le souligne le rapport Bur (2008), les entreprises qui versent à leurs salariés un $13^{\text {ème }}$ mois bénéficient d'un allègement plus élevé que celles qui octroient la même rémunération annuelle sur 12 mois. Cet effet d'aubaine n'apparaît pas dans la base de données DADS puisque le salaire horaire est calculé en fonction de la rémunération et du temps de travail annuels.

Afin de limiter les effets de cette déformation, on corrige la distribution de la main-d'œuvre au moyen d'un algorithme permettant d'obtenir un montant d'exonération plus proche de celui effectivement observé dans la base Acoss. Au terme de cette correction, la proportion de salariés dont la rémunération est inférieure à $1,1 \mathrm{Smic}$ a sensiblement augmenté (cf. tableau A). Cette augmentation est de 7 points pour les entreprises aux 39 heures et de 0,4 point pour les autres. Cette seconde distribution est plus cohérente avec d'autres études portant sur la distribution des rémunérations (Koubi et Lhommeau, 2007 ; Berry, 2008). D'après l'enquête Acémo de 2007

Tableau A

Distribution observée et corrigée de la main-d'œuvre par tranche de Smic

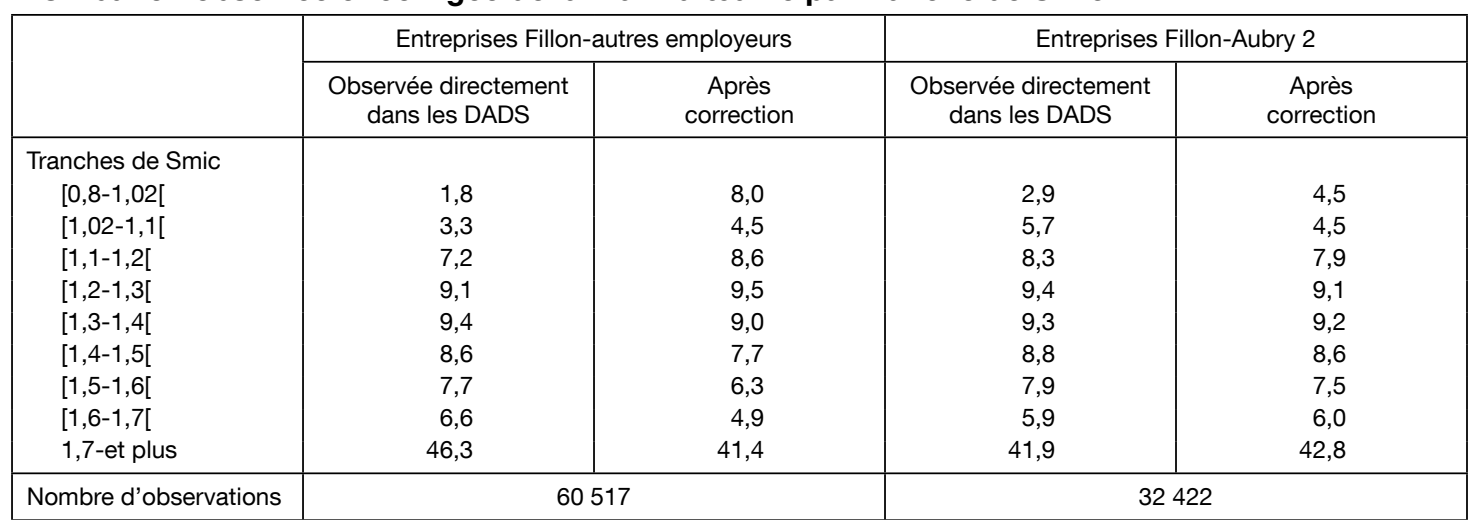

Champ : panel de 92939 entreprises de cinq salariés et plus au cours des années 2002 à 2005 appartenant aux secteurs d'activité privés non agricoles.

Source : bases Arome, Orme et Sequoia (Acoss), DADS et Suse (Insee).

Lecture : après correction de la distribution de la main d'œuvre par tranche de Smic, les entreprises Fillon-Aubry 2 comptent 42,8 \% de salariés rémunérés à un salaire supérieur à 1,7 Smic. 
environ $13 \%$ des salariés sont rémunérés sur la base du Smic. Les taux d'exonération sont ainsi obtenus au terme d'assez lourdes opérations de calcul et de redressement (cf. tableau B).

À partir du TEV obtenu pour le second semestre 2002, l'objectif est de calculer deux nouveaux taux pour le premier semestre de l'année 2005. Le premier s'appuie sur les barèmes d'allègements de cotisations sociales employeurs en vigueur en 2005 et sur la distribution des rémunérations observée en 2002, le second intègre simultanément ces barèmes ainsi que les hausses du Smic et des GMR prévues par la réforme Fillon. Le détail des calculs est le suivant :

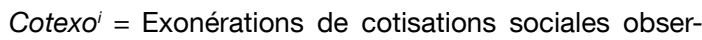
vées dans la base Acoss pour le semestre $i$ (02 pour le second semestre 2002 et 05 pour le premier semestre 2005).

Sal ${ }^{\prime}=$ Salaire brut observé dans la base Acoss pour le semestre $i$.

$\operatorname{Cotexo}\left(\widetilde{b^{i}}, d s a l^{02}\right)=$ Exonérations de cotisations socia-

les calculées à partir des barèmes légaux $\left(b^{\prime}\right)$ et de la distribution des salaires (dsal').

$\widetilde{S a l}^{02}=$ Salaire brut tenant compte de l'augmentation du Smic et de la GMR.

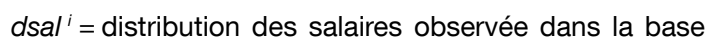
Dads.

$\widetilde{d s a l^{i}}=$ distribution des salaires corrigée.

TEA observé en $2002=\frac{\text { Cotexo }^{02}}{\text { Sal }^{02}}$

TEV-1 en 2002 sans correction sur la distribution des

salaires $=\frac{\operatorname{Cotexo}\left(\widetilde{b^{02}}, d s a l^{02}\right)}{S a l^{02}}$
TEV-2 en 2002 avec correction sur la distribution des salaires $=\frac{\operatorname{Cotexo}\left(\widetilde{b^{02}}, \widetilde{d s a l}{ }^{02}\right)}{S a l^{02}}$

TE observé en $2005=\frac{\text { Cotexo }^{05}}{S a l^{05}}$

TEV-2 en 2005 avec correction sur la distribution des salaires et augmentation du Smic et de la GMR = $\frac{\operatorname{Cotexo}\left(\widetilde{b^{05}}, \widetilde{d s a l}{ }^{02}\right)}{\widetilde{S a l}^{02}}$

\section{Évolution des TEV entre 2002 et 2005}

Comme nous l'avons déjà indiqué le TE observé a augmenté de plus de 2,5 points dans les entreprises aux 39 heures et a diminué de près de 1,5 points dans les entreprises aux 35 heures (cf. tableau B).

Le TEV calculé pour le second semestre 2002 est directement calé sur la distribution des salaires par tranche de Smic issue de la base DADS. II est parfois nettement plus faible que le TE observé, notamment pour les entreprises aux 39 heures (ligne 2 du tableau B). Or ces deux taux devraient en théorie être identiques, aux erreurs de mesure près.

En recalculant ces TE au moyen de la distribution des salaires par tranche de Smic corrigée, on obtient des résultats nettement plus satisfaisants, notamment pour les entreprises aux 39 heures (lignes 1 et 3 du tableau B). La comparaison des densités du TE observé et calculé avec et sans calage confirme que la correction effectuée améliore les résultats (les courbes représentant ces densités sont disponibles auprès des auteurs).

\section{Tableau B}

\section{Taux d'exonération observé et virtuel}

\begin{tabular}{|c|c|c|c|c|}
\hline & \multicolumn{2}{|c|}{ Entreprises aux 39 heures } & \multicolumn{2}{|c|}{ Entreprises aux 35 heures } \\
\hline & Sans pondération (3) & Avec pondération (3) & Sans pondération (3) & Avec pondération (3) \\
\hline \multicolumn{5}{|l|}{ En 2002} \\
\hline $\begin{array}{l}\text { TE observé } \\
\text { TEV sans correction (1) } \\
\text { TEV avec correction (1) }\end{array}$ & $\begin{array}{l}2,92 \\
1,89 \\
2,98\end{array}$ & $\begin{array}{l}2,01 \\
1,32 \\
2,06\end{array}$ & $\begin{array}{l}7,83 \\
8,87 \\
8,18\end{array}$ & $\begin{array}{l}5,78 \\
6,12 \\
6,00\end{array}$ \\
\hline \multicolumn{5}{|l|}{ En 2005} \\
\hline $\begin{array}{l}\text { TE observé } \\
\text { TEV avec correction (2) }\end{array}$ & $\begin{array}{l}7,18 \\
7,67\end{array}$ & $\begin{array}{l}4,52 \\
4,96\end{array}$ & $\begin{array}{l}6,52 \\
6,57\end{array}$ & $\begin{array}{l}4,34 \\
4,52\end{array}$ \\
\hline Nombre d'observations & \multicolumn{2}{|c|}{60517} & \multicolumn{2}{|c|}{32422} \\
\hline
\end{tabular}

Lecture : en 2002, et si l'on tient compte de la taille des entreprises (moyenne pondérée), le taux d'exonération observé moyen est de $2,01 \%$ dans les entreprises restées aux 39 heures.

Champ : panel de 92939 entreprises de cinq salariés et plus au cours des années 2002 à 2005 appartenant aux secteurs d'activité privés non agricoles.

Source : bases Arome, Orme et Sequoia (Acoss), DADS et Suse (Insee). 
ANNEXE 4

IMPACT DE LA LOI DE 2003 SUR LES SALAIRES ET L'EMPLOI

A - Pour les entreprises restées à 35 heures

\begin{tabular}{|c|c|c|c|c|c|c|c|}
\hline \multirow{2}{*}{$\begin{array}{l}\text { Structure } \\
(\text { en } \%)\end{array}$} & \multirow{2}{*}{$\begin{array}{l}\text { Variation du coût } \\
\text { du travail (en \%) }\end{array}$} & \multicolumn{2}{|c|}{$\mathrm{MCO}$} & \multicolumn{2}{|c|}{$\begin{array}{l}\text { Méthode du score-propension } \\
\text { (nearest neighbour) }\end{array}$} & \multicolumn{2}{|c|}{$\begin{array}{l}\text { Méthode du score-propension } \\
\text { (méthode du noyau) }\end{array}$} \\
\hline & & Coefficient & Écart-type & Coefficient & Écart-type & Coefficient & Écart-type \\
\hline \multicolumn{8}{|c|}{ Emploi (taux de croissance entre 2002 et 2005) } \\
\hline 15,5 & Inférieur à 0,5 & 0,0046 & 0,0042 & 0,007 & 0,006 & 0,006 & 0,004 \\
\hline 16,7 & Compris entre 0,5 et 1 & $-0,0002$ & 0,0041 & $0,012^{\star \star}$ & 0,006 & 0,009 & 0,005 \\
\hline 24,9 & Compris entre 1 et 2 & 0,0024 & 0,0038 & 0,01 & 0,006 & $0,014^{\star \star *}$ & 0,003 \\
\hline 21,7 & Compris entre 2 et 3 & $0,0087^{* *}$ & 0,0038 & $0,019^{\star * *}$ & 0,005 & $0,018^{\star * *}$ & 0,004 \\
\hline 21,1 & Supérieur à 3 & Réf. & - & Réf. & - & Réf. & - \\
\hline \multicolumn{8}{|c|}{ Emploi équivalent temps plein (taux de croissance entre 2002 et 2005) } \\
\hline 15,5 & Inférieur à 0,5 & $0,0135^{\star \star \star}$ & 0,0041 & $0,014^{\star *}$ & 0,006 & $0,017^{\star \star \star}$ & 0,005 \\
\hline 16,7 & Compris entre 0,5 et 1 & $0,0122^{\star \star \star}$ & 0,0041 & $0,023^{\star \star \star}$ & 0,006 & $0,020^{\star \star \star}$ & 0,004 \\
\hline 24,9 & Compris entre 1 et 2 & $0,0151^{\star \star \star}$ & 0,0038 & $0,020^{\star \star \star}$ & 0,006 & $0,022^{\star \star \star}$ & 0,003 \\
\hline 21,7 & Compris entre 2 et 3 & $0,0217^{\star \star \star}$ & 0,0037 & $0,031^{\star \star \star}$ & 0,005 & $0,030^{\star * *}$ & 0,004 \\
\hline 21,1 & Supérieur à 3 & Réf. & - & Réf. & - & Réf. & - \\
\hline \multicolumn{8}{|c|}{ Salaire horaire (taux de croissance entre 2002 et 2005) } \\
\hline 15,5 & Inférieur à 0,5 & 0,0043 & 0,0022 & 0,0030 & 0,0030 & 0,0050 & 0,004 \\
\hline 16,7 & Compris entre 0,5 et 1 & $-0,0002$ & 0,0022 & 0,0040 & 0,0030 & 0,0020 & 0,002 \\
\hline 24,9 & Compris entre 1 et 2 & $-0,0162^{\star \star \star}$ & 0,0021 & $-0,0130^{\star \star \star}$ & 0,0030 & $-0,0150^{\star \star \star}$ & 0,002 \\
\hline 21,7 & Compris entre 2 et 3 & $-0,0103^{\star \star \star}$ & 0,0020 & $-0,0050$ & 0,0030 & $-0,0070^{\star \star \star}$ & 0,002 \\
\hline 21,1 & Supérieur à 3 & Réf. & - & Réf. & - & Réf. & - \\
\hline
\end{tabular}

\section{B - Pour les entreprises restées à 39 heures}

\begin{tabular}{|c|c|c|c|c|c|c|c|}
\hline \multirow{2}{*}{$\begin{array}{l}\text { Structure } \\
\text { (en \%) }\end{array}$} & \multirow{2}{*}{$\begin{array}{l}\text { Variation du coût } \\
\text { du travail (en \%) }\end{array}$} & \multicolumn{2}{|c|}{ MCO } & \multicolumn{2}{|c|}{$\begin{array}{l}\text { Méthode du score-propension } \\
\text { (nearest neighbour) }\end{array}$} & \multicolumn{2}{|c|}{$\begin{array}{l}\text { Méthode du score-propension } \\
\text { (méthode du noyau) }\end{array}$} \\
\hline & & Coefficient & Écart-type & Coefficient & Écart-type & Coefficient & Écart-type \\
\hline \multicolumn{8}{|c|}{ Emploi (taux de croissance entre 2002 et 2005) } \\
\hline 8,6 & Supérieur à $+0,05$ & $-0,018^{\star \star \star}$ & 0,004 & $-0,014^{\star *}$ & 0,006 & $-0,016^{\star \star *}$ & 0,005 \\
\hline 5,0 & $\begin{array}{l}\text { Compris entre } \\
-0,03 \text { et }+0,05\end{array}$ & $-0,008^{\star \star}$ & 0,0039 & $0,015^{\star \star}$ & 0,007 & 0,008 & 0,005 \\
\hline 27,1 & $\begin{array}{l}\text { Compris entre } \\
-1 \text { et }-0,03\end{array}$ & $-0,010^{\star \star \star}$ & 0,0035 & 0,010 & 0,006 & 0,005 & 0,005 \\
\hline 26,5 & $\begin{array}{l}\text { Compris entre } \\
-2 \text { et }-1\end{array}$ & $-0,006^{\star}$ & 0,0032 & 0,002 & 0,005 & 0,003 & 0,004 \\
\hline 17,8 & $\begin{array}{l}\text { Compris entre } \\
-3 \text { et }-2\end{array}$ & $-0,008^{\star \star}$ & 0,0033 & $-0,005$ & 0,005 & $-0,003$ & 0,003 \\
\hline 15,0 & Inférieur à - 3 & Réf. & - & Réf. & - & Réf. & - \\
\hline \multicolumn{8}{|c|}{ Emploi équivalent temps plein (taux de croissance entre 2002 et 2005) } \\
\hline 8,6 & Supérieur à + 0,05 & $-0,0197^{\star * *}$ & 0,0042 & $-0,015^{\star \star \star}$ & 0,0060 & $-0,015^{\star \star \star}$ & 0,004 \\
\hline 5,0 & $\begin{array}{l}\text { Compris entre } \\
-0,03 \text { et }+0,05\end{array}$ & $-0,0033$ & 0,0038 & $-0,001$ & 0,0070 & 0,003 & 0,006 \\
\hline 27,1 & $\begin{array}{l}\text { Compris entre } \\
-1 \text { et }-0,03\end{array}$ & $-0,0105^{\star \star \star}$ & 0,0033 & $-0,007$ & 0,0060 & $-0,006$ & 0,004 \\
\hline 26,5 & $\begin{array}{l}\text { Compris entre } \\
-2 \text { et }-1\end{array}$ & $-0,0071^{\star \star}$ & 0,0031 & $-0,010^{\star \star}$ & 0,0050 & $-0,005$ & 0,004 \\
\hline 17,8 & $\begin{array}{l}\text { Compris entre } \\
-3 \text { et }-2\end{array}$ & $-0,0064^{\star \star}$ & 0,0032 & $-0,009^{\star \star}$ & 0,0046 & $-0,005$ & 0,004 \\
\hline 15,0 & Inférieur à - 3 & Réf. & - & Réf. & - & Réf. & - \\
\hline
\end{tabular}




\begin{tabular}{|c|c|c|c|c|c|c|c|}
\hline \multirow{2}{*}{$\begin{array}{l}\text { Structure } \\
\text { (en \%) }\end{array}$} & \multirow{2}{*}{$\begin{array}{l}\text { Variation du coût } \\
\text { du travail (en \%) }\end{array}$} & \multicolumn{2}{|c|}{$\mathrm{MCO}$} & \multicolumn{2}{|c|}{$\begin{array}{l}\text { Méthode du score-propension } \\
\text { (nearest neighbour) }\end{array}$} & \multicolumn{2}{|c|}{$\begin{array}{l}\text { Méthode du score-propension } \\
\text { (méthode du noyau) }\end{array}$} \\
\hline & & Coefficient & Écart-type & Coefficient & Écart-type & Coefficient & Écart-type \\
\hline \multicolumn{8}{|c|}{ Salaire horaire (taux de croissance entre 2002 et 2005) } \\
\hline 8,6 & Supérieur à $+0,05$ & $-0,0203^{\star * *}$ & 0,0023 & $-0,014^{\star \star \star}$ & 0,003 & $-0,015^{\star \star \star}$ & 0,0027 \\
\hline 5,0 & $\begin{array}{l}\text { Compris entre }-0,03 \\
\text { et }+0,05\end{array}$ & $-0,0367^{\star \star \star}$ & 0,0022 & $-0,039^{\star \star \star}$ & 0,004 & $-0,042^{\star \star \star}$ & 0,0043 \\
\hline 27,1 & $\begin{array}{l}\text { Compris entre }-1 \text { et } \\
-0,03\end{array}$ & $-0,0319^{\star \star \star}$ & 0,0019 & $-0,038^{\star \star \star}$ & 0,003 & $-0,037^{\star \star \star}$ & 0,0035 \\
\hline 26,5 & $\begin{array}{l}\text { Compris entre }-2 \\
\text { et }-1\end{array}$ & $-0,0218^{\star \star \star}$ & 0,0016 & $-0,024^{\star \star \star}$ & 0,002 & $-0,026^{\star \star \star}$ & 0,0024 \\
\hline 17,8 & $\begin{array}{l}\text { Compris entre - } 3 \\
\text { et }-2\end{array}$ & $-0,0129^{\star \star \star}$ & 0,0017 & $-0,015^{\star \star \star}$ & 0,002 & $-0,015^{\star \star \star}$ & 0,0025 \\
\hline 15,0 & Inférieur à - 3 & Réf. & - & Réf. & - & Réf. & - \\
\hline
\end{tabular}

Lecture : les estimations sont obtenues sous STATA (programmes de Sianesi et Leuven (2003)). Les variances sont calculées par la méthode du bootstrap (50 tirages). *** : significatif au seuil de $1 \% ;{ }^{* *}: 5 \% ;{ }^{*}: 10 \%$.

Tableau A : les entreprises passées aux 35 heures avant 2003 et qui ont expérimenté une hausse du coût du travail inférieure à $0,5 \%$ ont vu leur niveau d'emploi en équivalent temps plein augmenter $(+0,0135)$ par rapport aux entreprises aux 35 heures dans lesquelles le coût du travail s'est accru de plus de 3 \%. L'interprétation des coefficient du tableau B est similaire.

Champ : panel de 92939 entreprises de cinq salariés et plus au cours des années 2002 à 2005 appartenant aux secteurs d'activité privés non agricoles.

Source : bases Arome, Orme et Sequoia (Acoss), DADS et Suse (Insee). 
EFFET DE LA LOI DE 2003 SUR LE TAUX DE CROISSANCE DE L'EMPLOI (ESTIMATION PAR LA MÉTHODE MCO)

\begin{tabular}{|c|c|c|c|c|}
\hline & \multicolumn{2}{|c|}{ Entreprises passées à 35 heures } & \multicolumn{2}{|c|}{ Entreprises restées à 39 heures } \\
\hline & Coefficient & Écart-type & Coefficient & Écart-type \\
\hline $\begin{array}{l}\text { Informations sectorielles } \\
\text { Industrie } \\
\text { Construction } \\
\text { Commerce } \\
\text { Autres services } \\
\text { Taux d'entrée } \\
\text { Taux de sortie } \\
\text { Indice d'Herfindhal (1) }\end{array}$ & $\begin{array}{l}-0,0273^{\star \star \star} \\
-0,0022 \\
-0,0104^{\star \star \star} \\
\\
0,1763^{\star \star \star} \\
-0,0684^{\star} \\
-0,0285\end{array}$ & $\begin{array}{l}0,0054 \\
0,0051 \\
0,0039 \\
0,0366 \\
0,0377 \\
0,0194\end{array}$ & $\begin{array}{c}-0,0335^{\star \star \star} \\
-0,0084^{\star \star \star} \\
-0,0204^{\star \star \star} \\
0,1035^{\star \star \star} \\
-0,0917^{\star \star \star} \\
0,0124\end{array}$ & $\begin{array}{l}0,0034 \\
0,0032 \\
0,0029 \\
0,0279 \\
0,0300 \\
0,0200\end{array}$ \\
\hline $\begin{array}{l}\text { Autres caractéristiques } \\
\text { Localisation en île de France } \\
\text { Appartenance à un groupe } \\
\text { Log (Intensité capitalistique) }\end{array}$ & $\begin{array}{l}-0,0152^{\star \star \star} \\
0,0085^{\star \star} \\
0,0012\end{array}$ & $\begin{array}{l}0,0041 \\
0,0042 \\
0,0015\end{array}$ & $\begin{array}{r}-0,0191^{\star \star \star} \\
0,0123^{\star \star \star} \\
0,0048^{\star \star \star}\end{array}$ & $\begin{array}{l}0,0025 \\
0,0034 \\
0,0012\end{array}$ \\
\hline $\begin{array}{l}\text { Caractéristiques de la main d'œuvre } \\
\text { Effectif brut en } 2002 \\
\text { Grandes entreprises (50 salariés et plus) } \\
\text { Part des femmes } \\
\text { Part des salariés à temps partiel } \\
\text { Emplois peu qualifiés (au moins } 30 \% \\
\text { de la main d'œuvre) } \\
\text { Emplois très qualifiés (au moins } 20 \% \\
\text { de la main d'œuvre) } \\
\text { Log (facteur de croissance de l'effectif } \\
\text { entre } 2000 \text { et 2002) }\end{array}$ & $\begin{array}{r}-0,0051 \\
0,0227^{\star \star \star} \\
-0,0174^{\star \star \star} \\
0,0541^{\star \star \star} \\
-0,0172^{\star \star \star} \\
0,0088^{\star \star \star} \\
0,0580^{\star \star \star}\end{array}$ & $\begin{array}{l}0,0029 \\
0,0043 \\
0,0056 \\
0,0059 \\
0,0028 \\
0,0027 \\
0,0069\end{array}$ & $\begin{array}{r}-0,0148^{\star \star \star} \\
0,0304^{\star \star \star} \\
-0,0324^{\star \star \star} \\
0,0620^{\star \star \star} \\
-0,0185^{\star \star \star} \\
0,0065^{\star \star \star} \\
0,0562^{\star \star \star}\end{array}$ & $\begin{array}{l}0,0055 \\
0,0033 \\
0,0046 \\
0,0051 \\
0,0023 \\
0,0020 \\
0,0050\end{array}$ \\
\hline $\begin{array}{l}\text { Variables croisées } \\
\text { Industrie*(effectif brut }>50 \text { salariés) } \\
\text { Industrie*appartenance à un groupe }\end{array}$ & $\begin{array}{l}-0,0264^{\star \star \star} \\
-0,0072\end{array}$ & $\begin{array}{l}0,0061 \\
0,0063\end{array}$ & $\begin{array}{l}-0,0217^{\star \star \star} \\
-0,0014\end{array}$ & $\begin{array}{l}0,0046 \\
0,0055\end{array}$ \\
\hline $\begin{array}{l}\text { Variables comptables et financières } \\
\text { Niveau d'endettement en } 2002 \\
\text { Rentabilité économique en } 2002 \\
\text { Taux de marge en } 2002 \\
\text { Taux de croissance du coût du travail } \\
\text { entre } 2000 \text { et } 2002 \\
\text { Log (productivité apparente du travail en 2002) } \\
\text { Log (facteur de croissance du coût du travail } \\
\text { généré par les lois Fillon) }\end{array}$ & $\begin{array}{l}-0,0047 \\
0,0469^{\star \star \star} \\
0,1763^{\star \star \star} \\
0,0812^{\star \star \star} \\
-0,0006 \\
-0,2776^{\star \star \star}\end{array}$ & $\begin{array}{l}0,0087 \\
0,0054 \\
0,0109 \\
0,0071 \\
0,0017 \\
0,0931\end{array}$ & $\begin{array}{c}0,0018 \\
0,0514^{\star \star \star} \\
0,1616^{\star \star \star} \\
0,0645^{\star \star \star} \\
-0,0041^{\star \star} \\
-0,2915^{\star \star \star}\end{array}$ & $\begin{array}{l}0,0076 \\
0,0041 \\
0,0094 \\
0,0054 \\
0,0016 \\
0,0584\end{array}$ \\
\hline Constante & $-0,0547^{\star \star \star}$ & 0,0127 & $-0,0330^{\star \star \star}$ & 0,0117 \\
\hline
\end{tabular}

Lecture : on prend en compte un grand nombre de variables de façon à estimer des effets sur l'emploi toutes choses égales par ailleurs. C'est le cas en particulier des variables croisées qui permettent de contrôler les effets au sein de chaque secteur en fonction de la taille ou de l'appartenance à un groupe.

Champ : panel de 92939 entreprises de cinq salariés et plus au cours des années 2002 à 2005 appartenant aux secteurs d'activité privés non agricoles.

Source : bases Arome, Orme et Sequoia (Acoss), DADS et Suse (Insee). 


\section{SPÉCIFICATION DES SCORES DE PROPENSION (ESTIMATION DU MODĖLE PROBIT ORDONNÉ)}

\begin{tabular}{|c|c|c|c|c|}
\hline & \multicolumn{2}{|c|}{ Entreprises aux 35 heures } & \multicolumn{2}{|c|}{ Entreprises aux 39 heures } \\
\hline & Coefficient & Écart-type & Coefficient & Écart-type \\
\hline \multicolumn{5}{|l|}{ Informations sectorielles } \\
\hline Industrie & $-0,1632^{\star \star \star}$ & 0,0261 & $-0,156^{\star \star \star}$ & 0,016 \\
\hline Construction & $0,5920^{\star \star \star}$ & 0,0241 & $-0,753^{\star \star \star}$ & 0,015 \\
\hline Commerce & 0,0269 & 0,0192 & $-0,198^{\star \star \star}$ & 0,013 \\
\hline Autres services & & & & \\
\hline Taux d'entrée & $-0,2947^{\star}$ & 0,1665 & $-2,341^{\star \star \star}$ & 0,120 \\
\hline Taux de sortie & $0,7052^{\star \star \star}$ & 0,1851 & $1,512^{\star \star \star}$ & 0,140 \\
\hline Indice d'Herfindhal (1) & $-0,4161^{\star \star \star}$ & 0,1055 & $-0,953^{\star \star \star}$ & 0,091 \\
\hline \multicolumn{5}{|l|}{ Autres caractéristiques } \\
\hline Localisation en lle de France & $-0,2831^{\star \star \star}$ & 0,0160 & $-0,362^{\star \star \star}$ & 0,010 \\
\hline Appartenance à un groupe & $-0,1313^{\star \star \star}$ & 0,0199 & $-0,082^{\star \star \star}$ & 0,014 \\
\hline Log (Intensité capitalistique) & $-0,0451^{\star \star \star}$ & 0,0071 & 0,002 & 0,005 \\
\hline \multicolumn{5}{|l|}{ Caractéristiques de la main d'œuvre } \\
\hline Effectif brut en 2002 & $0,1327^{\star \star \star}$ & 0,0485 & $-0,221^{\star \star \star}$ & 0,054 \\
\hline Grandes entreprises (50 salariés et plus) & $-0,1496^{\star \star \star}$ & 0,0206 & 0,022 & 0,014 \\
\hline Part des femmes & $0,2425^{\star \star \star}$ & 0,0278 & $-0,591^{\star \star \star}$ & 0,023 \\
\hline Part des salariés à temps partiel & $-0,0284$ & 0,0311 & $-0,611^{\star \star \star}$ & 0,026 \\
\hline $\begin{array}{l}\text { Emplois peu qualifiés (au moins } 30 \% \text { de la main } \\
\text { d'œuvre) }\end{array}$ & $-0,1147^{\star \star \star}$ & 0,0149 & $-0,055^{\star \star \star}$ & 0,011 \\
\hline $\begin{array}{l}\text { Emplois très qualifiés (au moins } 20 \text { \% de la main } \\
\text { d'œuvre) }\end{array}$ & $-0,0074$ & 0,0142 & $-0,358^{\star \star *}$ & 0,010 \\
\hline $\begin{array}{l}\text { Log (facteur de croissance de l'effectif entre } 2000 \\
\text { et 2002) }\end{array}$ & $0,1080^{\star \star \star}$ & 0,0317 & $-0,018$ & 0,022 \\
\hline \multicolumn{5}{|l|}{ Variables croisées } \\
\hline Industrie ${ }^{\star}$ (effectif brut >50 salariés) & $0,2484^{\star \star \star}$ & 0,0295 & $-0,118^{\star \star \star}$ & 0,020 \\
\hline Industrie*appartenance à un groupe & 0,0434 & 0,0300 & 0,000 & 0,023 \\
\hline \multicolumn{5}{|l|}{ Variables comptables et financières } \\
\hline Niveau d'endettement en 2002 & $0,3201^{\star \star \star}$ & 0,0420 & $0,196^{\star \star \star}$ & 0,036 \\
\hline Rentabilité économique en 2002 & $-0,1420^{\star \star \star}$ & 0,0243 & $-0,032$ & 0,017 \\
\hline Taux de marge en 2002 & $0,5109^{\star \star \star}$ & 0,0532 & $0,169^{\star \star \star}$ & 0,042 \\
\hline $\begin{array}{l}\text { Taux de croissance du coût du travail entre } 2000 \\
\text { et } 2002\end{array}$ & $0,0890^{\star \star \star}$ & 0,0332 & $0,050^{\star *}$ & 0,024 \\
\hline Log (productivité apparente du travail en 2002) & $-0,0276^{\star \star \star}$ & 0,0083 & $-0,058^{\star \star \star}$ & 0,007 \\
\hline Constante 1 & $-1,2093^{\star \star \star}$ & 0,0645 & $-2,742^{\star \star \star}$ & 0,054 \\
\hline Constante 2 & $-0,6443^{\star \star \star}$ & 0,0643 & $-2,165^{\star \star \star}$ & 0,053 \\
\hline Constante 3 & 0,0245 & 0,0642 & $-1,552^{\star \star \star}$ & 0,052 \\
\hline Constante 4 & $0,6798^{\star \star \star}$ & 0,0642 & $-0,807^{\star \star \star}$ & 0,052 \\
\hline Constante 5 & - & - & $-0,149^{\star \star \star}$ & 0,052 \\
\hline Nombre d'observations & \multicolumn{2}{|c|}{32422} & \multicolumn{2}{|c|}{60517} \\
\hline
\end{tabular}

Lecture : on prend en compte un grand nombre de variables de façon à estimer des effets sur l'emploi toutes choses égales par ailleurs. C'est le cas en particulier des variables croisées qui permettent de contrôler les effets au sein de chaque secteur en fonction de la taille ou de l'appartenance à un groupe.

Champ : panel de 92939 entreprises de cinq salariés et plus au cours des années 2002 à 2005 appartenant aux secteurs d'activité privés non agricoles.

Source : bases Arome, Orme et Sequoia (Acoss), DADS et Suse (Insee). 


\section{Où en est l'insertion professionnelle?}

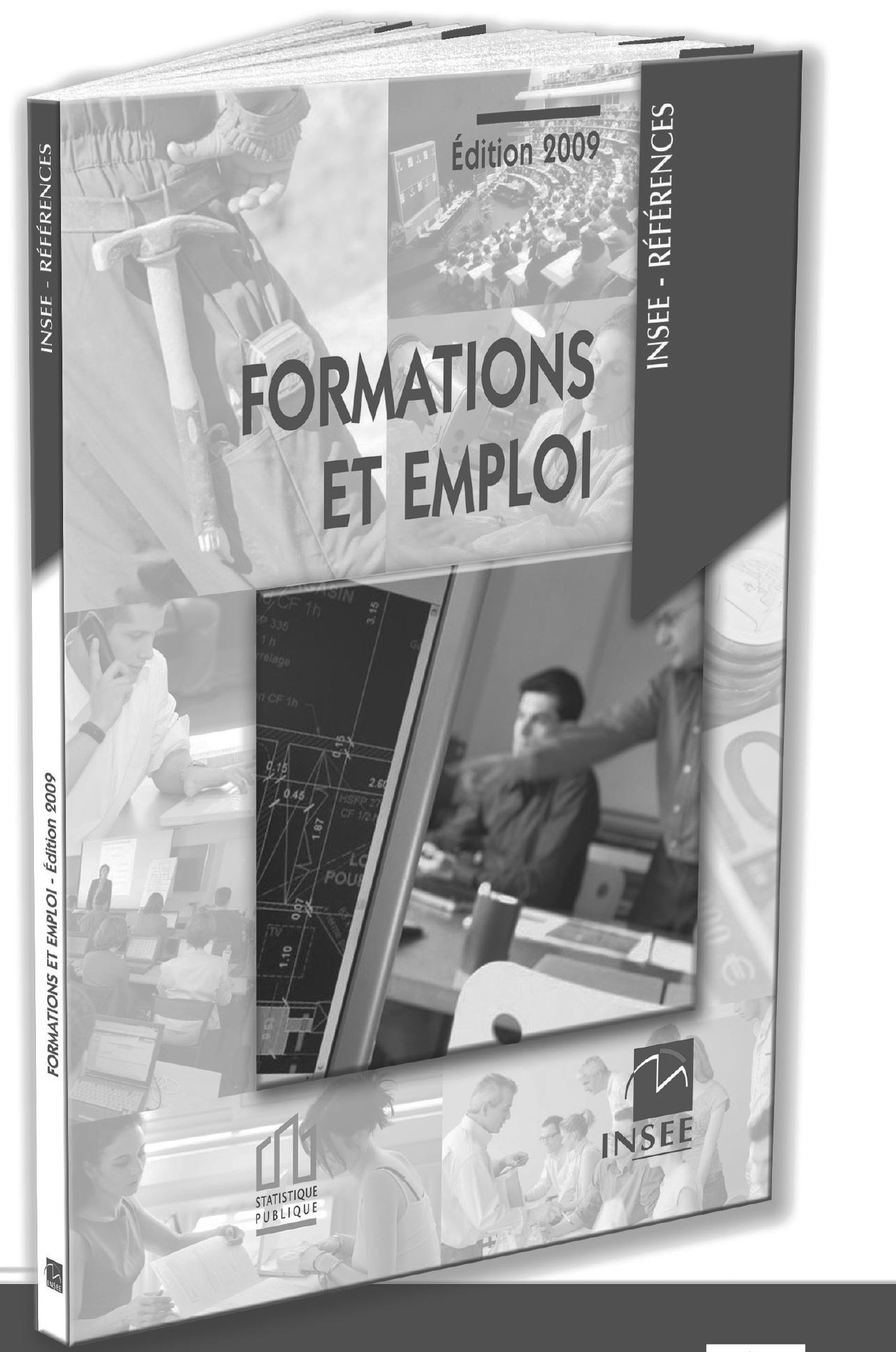

En vente dans les librairies, par correspondance et sur www.insee.fr

- I'influence de la formation continue sur l'accès à la promotion sociale,

- la correspondance entre les spécialités de formation et les domaines d'emploi,

- l'apprentissage, entre formation et insertion professionnelles. 